DOI 10.4171/JEMS/296

Artur Avila $\cdot$ Jairo Bochi · David Damanik

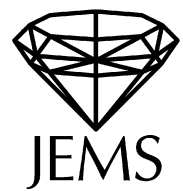

\title{
Opening gaps in the spectrum of strictly ergodic Schrödinger operators
}

Received March 16, 2010

\begin{abstract}
We consider Schrödinger operators with dynamically defined potentials arising from continuous sampling along orbits of strictly ergodic transformations. The Gap Labeling Theorem states that the possible gaps in the spectrum can be canonically labelled by an at most countable set defined purely in terms of the dynamics. Which labels actually appear depends on the choice of the sampling function; the missing labels are said to correspond to collapsed gaps. Here we show that for any collapsed gap, the sampling function may be continuously deformed so that the gap immediately opens. As a corollary, we conclude that for generic sampling functions, all gaps are open. The proof is based on the analysis of continuous $\operatorname{SL}(2, \mathbb{R})$ cocycles, for which we obtain dynamical results of independent interest.
\end{abstract}

\section{Contents}

1. Introduction ....... 62

2. Statement of further results for $\operatorname{SL}(2, \mathbb{R})$-cocycles $\ldots \ldots \ldots \ldots \ldots$

3. Outline of the paper and discussion of the methods . . . . . . . . . . . . . . 72

4. Construction of almost invariant sections . . . . . . . . . . . . . . . . . . 73

5. Construction of sets with controlled return times . . . . . . . . . . . . 76

6. From slow growth to invariant sections for the disk action . . . . . . . . . . . . . . 78

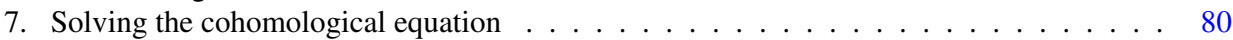

8. Extending families of cocycles: small Lyapunov exponents . . . . . . . . . . . . . . 83

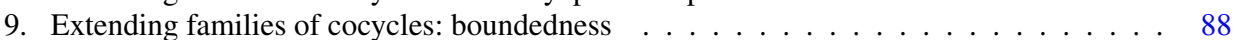

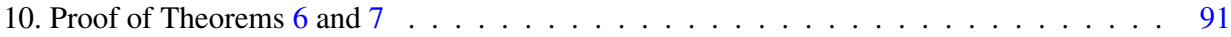

Appendix A. Connectedness considerations . . . . . . . . . . . . . . . . . . . . . . . . . 94

Appendix B. Minimal examples with many persistently closed gaps _ . . . . . . . . . . . . . 97

Appendix C. Uniform hyperbolicity and the rotation number . . . . . . . . . . . . . . . 98

Appendix D. Proof of the projection lemma . . . . . . . . . . . . . . . . . . . . 101

Appendix E. Mixing dynamics and Cantor spectrum . . . . . . . . . . . . . . . . . . . 102

References . . . . . . . . . . . . . . . . . . . . . . . . 103

A. Avila: CNRS UMR 7599, Laboratoire de Probabilités et Modèles aléatoires, Université Pierre et Marie Curie, Boîte courrier 188, 75252 Paris Cedex 05, France; e-mail: artur@ math.sunysb.edu, URL: www.impa.br/ avila

J. Bochi: Departamento de Matemática, PUC-Rio, Rua Marquês de São Vicente, 225, 22453-900 Rio de Janeiro, RJ, Brazil; e-mail: jairo@ mat.puc-rio.br, URL: www.mat.puc-rio.br/ jairo

D. Damanik: Department of Mathematics, Rice University, Houston, TX 77005, USA; e-mail: damanik@ rice.edu, URL: www.ruf.rice.edu/ dtd3 


\section{Introduction}

\subsection{Gap opening}

Let $X$ be a compact metric space and let $f: X \rightarrow X$ be a strictly ergodic homeomorphism (hence all orbits are dense and equidistributed with respect to an invariant probability measure $\mu$ ). We will also assume that $f$ has a non-periodic finite-dimensional factor. ${ }^{1}$

Here we are interested in the one-dimensional discrete Schrödinger operator $H=$ $H_{f, v, x}$ whose potential is obtained by continuous sampling along an orbit of $f$. Thus $H$ is a bounded self-adjoint operator on $\ell^{2}(\mathbb{Z})$ given by

$$
[H \psi](n)=\psi(n+1)+\psi(n-1)+V(n) \psi(n),
$$

where the potential $V$ is given by $V(n)=v\left(f^{n}(x)\right)$ for some $x \in X$ and some continuous sampling function $v: X \rightarrow \mathbb{R}$.

Since $f$ is minimal, the spectrum of $H$ turns out to depend only on $f$ and $v$, but not on $x$, and hence may be denoted by $\Sigma_{f, v}$; it is a perfect compact subset of $\mathbb{R}$. Strict ergodicity implies that the integrated density of states is well defined and only depends on $f$ and $v$ : it is a continuous non-decreasing surjective function $N_{f, v}: \mathbb{R} \rightarrow[0,1]$. (See $\S 1.5$ for details.) The basic relation between $\Sigma_{f, v}$ and $N_{f, v}$ is that $E \notin \Sigma_{f, v}$ if and only if $N_{f, v}$ is constant in a neighborhood of $E$. Hence, in each bounded connected component of $\mathbb{R} \backslash \Sigma_{f, v}$ (called a gap of $\Sigma_{f, v}$ ), $N_{f, v}$ assumes a constant value $\ell \in(0,1)$, called the label of the gap. A basic question is thus: which values of $\ell$ appear as labels of gaps?

There is a well known general partial answer to this problem known as the Gap Labeling Theorem. Let $G \subset \mathbb{R}$ be the subgroup of all $t$ such that there exist continuous maps $\phi: X \rightarrow \mathbb{R}$ and $\psi: X \rightarrow \mathbb{R} / \mathbb{Z}$ with $t=\int \phi d \mu$ and $\psi(f(x))-\psi(x)=\phi(x) \bmod 1$. Thus $G$ only depends on $f$ and it is called the range of the Schwartzman asymptotic cycle. ${ }^{2}$ Often $G$ is a non-discrete group: this happens for instance whenever $X$ has infinitely many connected components.

Gap Labeling Theorem. If $\ell$ is the label of some gap, then $\ell \in \mathcal{L}=G \cap(0,1)$.

Of course, the Gap Labeling Theorem does not guarantee that any particular $\ell \in \mathcal{L}$ actually is the label of some gap: it is quite easy to see that the specific choice of the sampling function is also important. A missing label, that is, some $\ell \in \mathcal{L}$ which is not the label of an actual gap, is usually said to correspond to the collapsed gap $N_{f, v}^{-1}(\ell)$ (actual gaps are sometimes called open gaps for this reason).

Describing which gaps are open in specific situations (and in particular, whether the converse of the Gap Labeling Theorem holds) is usually a very difficult problem. The well known "Dry Ten Martini Problem" (cf. [Si2]) is an instance of this: it asks one to show that all gaps are open for the almost Mathieu operator $(X=\mathbb{R} / \mathbb{Z}, f(x)=x+\alpha$ for some $\alpha \in \mathbb{R} \backslash \mathbb{Q}$ and $v(x)=2 \lambda \cos 2 \pi x$ for some $\lambda>0$ ). This problem is still open, despite considerable attention (for partial progress, see [CEY], [Pu], [AJ1], and [AJ2]).

\footnotetext{
1 That is, there is a homeomorphism $g: Y \rightarrow Y$, where $Y$ is an infinite compact subset of some Euclidean space $\mathbb{R}^{d}$, and there is an onto continuous map $h: X \rightarrow Y$ such that $h \circ f=g \circ h$.

2 See [Sc], [Pa, §5.2], [At]; Theorem 4.2 from [At] justifies this terminology.
} 
Here we are concerned with the more general problem of whether the dynamics, by itself, could force the closing of (many) gaps. Questions in this direction arose early in the development of the theory: in a 1982 paper, Belissard asks whether the mixing property would prevent the set of labels of open gaps to be dense in $(0,1)$ (or equivalently, whether "mixing avoids Cantor spectrum" [Be1, Open Problem 5]).

We will show that the dynamics in fact does not obstruct the opening of gaps, and indeed the converse of the Gap Labeling Theorem holds for generic sampling functions. This is obtained as a consequence of the following result, which shows that any collapsed gap can be opened by deformation:

Theorem 1 (Gap opening). For every $v \in C(X, \mathbb{R})$ and any $\ell \in \mathcal{L}$, there exists a continuous path $v_{t} \in C(X, \mathbb{R}), 0 \leq t \leq 1$ with $v_{0}=v$ such that for every $t>0$, the spectrum $\Sigma_{f, v_{t}}$ has an open gap with label $\ell$.

Since the sampling functions for which the gap with label $\ell$ is open form an open subset of $C(X, \mathbb{R})$, we have the following:

Corollary 2 (Generic converse to the Gap Labeling Theorem). For a generic $v \in$ $C(X, \mathbb{R})$, all gaps are open, that is, for every gap label $\ell \in \mathcal{L}$, the spectrum $\Sigma_{f, v}$ has an open gap with label $\ell$.

In particular, this gives, in our setting, an affirmative answer to another question of Bellissard, who asked in [Be2, Question 4] whether Cantor spectrum is generic whenever it is not precluded by the Gap Labeling Theorem. (A specific mixing example falling in this category is discussed below, answering his earlier question as well.) On the other hand, as we will show in Appendix B, the answer to Bellissard's question becomes negative if one considers slightly more general base dynamics $f$.

Remark 1.1. We do not know whether for $v \in C(X, \mathbb{R})$ given, it is possible to find a continuous path $v_{t} \in C(X, \mathbb{R}), 0 \leq t \leq 1$, with $v_{0}=v$ and such that for every $t>0$ all gaps of $\Sigma_{f, v_{t}}$ are open. ${ }^{3}$

\subsection{Examples}

One important class of examples (which includes the almost Mathieu operator) is given by almost periodic Schrödinger operators, for which $X$ is an infinite compact Abelian group and $f$ is a minimal translation: in this case $\mathcal{L}$ is a dense subset of $(0,1)$. Two important subclasses are given by quasi-periodic Schrödinger operators, where $X=\mathbb{R}^{d} / \mathbb{Z}^{d}$ for some $d \geq 1$, and by limit-periodic Schrödinger operators, where $X$ is a product of finite cyclic groups.

If $\mathcal{L}$ is not dense in $(0,1)$, it is impossible for $\Sigma_{f, v}$ to be nowhere dense. On the other hand, when $\mathcal{L}$ is dense in $(0,1)$, particularly in the almost periodic case, much effort has been dedicated to establishing Cantor spectrum (which means that a dense set of gaps are

\footnotetext{
3 The difficulty is that we deduce Theorem 1 from Theorem 3, and that result does not hold with an extra parameter-see Remark A.4.
} 
open). For limit-periodic Schrödinger operators, this issue was studied by various authors in the 1980's (e.g., [M], [AS1], [Ch], [PT]) and, in fact, Corollary 2 is known in this particular case (see [Si1] for an earlier account in the periodic case). However, already for quasi-periodic Schrödinger operators, the problem is much harder.

For the almost Mathieu operator, proving Cantor spectrum corresponds to the Ten Martini Problem, which is now completely solved [AJ1], after several partial results ([BSi], [HS], [CEY], [L], [Pu], [AK]). More generally, the case of non-constant analytic sampling functions along translations of tori has been considered, first by KAM-like methods and more recently by non-perturbative approaches. For Diophantine translations on tori, in any dimension, Eliasson [E] has shown that for sufficiently small generic analytic potentials, all gaps are open. For general non-constant analytic sampling functions $v$, Goldstein and Schlag [GS] showed that a dense set of gaps can be opened "almost surely" by changing $\alpha$, in the case where the Lyapunov exponent is positive (which includes the case of large $v$ ).

Previous to this work, we showed in $[\mathrm{ABD}]^{4}$ that generically, a dense set of gaps are open, but only for a more restricted set of dynamics ("generalized skew-shifts"): those are the dynamics which fiber over an almost periodic dynamical system. They also have dense $\mathcal{L}$. An example in this class of particular interest is the skew-shift $(x, y) \mapsto$ $(x+\alpha, y+x)$. However, generalized skew-shifts still form a somewhat restricted class; for instance, they are never weakly mixing. Moreover, the work [ABD] was not able to control the labels of the open gaps produced and in particular could not guarantee the opening of a gap with a prescribed label.

With respect to mixing properties, we would like to mention Fayad's analytic map $f$ on $X=\mathbb{R}^{3} / \mathbb{Z}^{3}$ from [Fa] which is strictly ergodic, mixing (hence not a generalized skew-shift), and has dense $\mathcal{L} .^{5}$ Applying our results to this example yields, to the best of our knowledge, the first example of Cantor spectrum for Schrödinger operators arising from mixing dynamics. Of course, an interesting example of a strictly ergodic dynamical system is given by the time one map of the horocycle flow for a compact hyperbolic surface. This is again mixing, but in this case $\mathcal{L}$ is empty!

Other interesting examples of "strictly ergodic dynamics" which are weakly mixing are given by typical interval exchange transformations [AF]. They are not actually strictly ergodic according to the usual definition since they are not homeomorphisms, but this can be bypassed by going to a symbolic setting (blowing up procedure; see [MMY]).

Of course, in the symbolic context, and more generally, among Cantor set maps, there is a wealth of examples, and all have dense $\mathcal{L}$. See also [BCL] for related examples of homeomorphisms of manifolds.

Remark 1.2. In the analytic setting, it is far from clear whether one should expect that the generic converse of the Gap Labeling Theorem, or even generic Cantor spectrum, remains true for translations on tori in higher (at least 2) dimensions; compare [CS]. We do expect it to be true in one dimension, which is supported by recent advances such as

\footnotetext{
4 Results related to [ABD] can be found in the papers [CF], [FJ], [FJP].

5 Details are provided in Appendix E.
} 
[AJ2] and [GS], but despite this progress, it is not yet known whether the "analytically generic" spectrum is a Cantor set (even restricting to Diophantine translations).

\subsection{A brief history of gap labeling}

Gap labeling theorems were first established in the early 1980's. In 1982, Johnson and Moser [JM] considered continuum Schrödinger operators, $H=-d^{2} / d x^{2}+V$, with almost periodic potential $V$. For $E \in \mathbb{R}$, if $u=u(x, E)$ is a non-trivial real solution of the associated differential equation $-u^{\prime \prime}+V u=E u$, then $u^{\prime}(x, E)+i u(x, E)$ never vanishes; this allowed them to define the rotation number by

$$
\rho(E)=\lim _{x \rightarrow \infty} \frac{1}{x} \arg \left(u^{\prime}(x, E)+i u(x, E)\right) .
$$

They showed that the limit exists, is independent of the solution chosen, and defines a continuous non-decreasing function of $E$. They also pointed out that due to Sturm oscillation theory, there is a simple relation between the rotation number and the integrated density of states. Since $V$ is almost periodic, $M_{V}(\lambda)=\lim _{x \rightarrow \infty} x^{-1} \int_{0}^{x} V(y) e^{-i \lambda y} d y$ exists for every $\lambda \in \mathbb{R}$ and there are at most countably many $\lambda$ 's for which $M_{V}(\lambda) \neq 0$. The set $\mathcal{M}(V)$ of finite integer linear combinations of these $\lambda$ 's is called the frequency module of $V$. Johnson and Moser then showed that the function $\rho$ is constant precisely on the complement of the spectrum $H$ and, moreover, if $E \in \mathbb{R} \backslash \sigma(H)$, then $2 \rho(E) \in \mathcal{M}(V)$. Thus, one can label the gaps of the spectrum by elements of the frequency module of $V$.

The analogue of the Johnson-Moser results for ergodic discrete Schrödinger operators (and Jacobi matrices) can be found in the 1983 paper [DS] by Delyon and Souillard. In essence, they used Sturm oscillation theory and the easy existence proof for the integrated density of states to define the rotation number in their setting. The relation between the rotation number and the integrated density of states is therefore built into this approach and continues to hold trivially. Moreover, in the case of almost periodic potential, the gaps in the spectrum may again be labeled by elements of the frequency module of the potential.

Around the same time, Bellissard, Lima, and Testard began to develop an approach to gap labeling based on $K$-theory of $C^{*}$-algebras; see [Be1] for the earliest account and $[\mathrm{Be} 2, \mathrm{Be} 3, \mathrm{Be} 4, \mathrm{BLT}]$ for extensive discussions. A major advantage of this approach is that it is not restricted to one space dimension. Let us discuss it in the one-dimensional discrete setting relevant to our present study, but emphasize that the results hold in greater generality. Given a compact space $X$ and a homeomorphism $f$ of $X$, one can define an associated $C^{*}$-algebra $C^{*}(X, f)$. An $f$-ergodic probability measure $\mu$ induces a trace $\tau$ on $C^{*}(X, f)$. Consider the $K_{0}$ group associated with $C^{*}(X, f)$ and the induced map $\tau_{*}$ : $K_{0}\left(C^{*}(X, f)\right) \rightarrow \mathbb{R}$. The abstract gap labeling theorem now reads as follows: Suppose $H$ is a self-adjoint operator affiliated to $C^{*}(X, f)$ such that its integrated density of states exists with respect to the $f$-ergodic measure $\mu$. Then the value of the integrated density of states in a gap of the spectrum belongs to $\tau_{*}\left(K_{0}\left(C^{*}(X, f)\right)\right) \cap(0,1)$. This set of labels is not quite easy to compute, in general. For the case of an irrational rotation of the circle, this was accomplished using the Pimsner-Voiculescu cyclic six-term exact 
sequence [PV]. This and other computational aspects of the theory are discussed in the references given above. In this context, we would like to also mention the paper $[\mathrm{X}]$ by $\mathrm{Xia}$, which shows that the integers in the quasi-periodic gap labeling can be expressed as Chern characters.

The direct relation between taking the image under the trace homomorphism of the $K_{0}$ group of $C^{*}(X, f)$ and taking the image under Schwartzman's asymptotic cycle of the first Cech cohomology group $\check{H}^{1}\left(Y_{f}, \mathbb{Z}\right)$, where $Y_{f}$ is the suspension space of $(X, f)$, was pointed out by Johnson [J] in 1986; compare also Connes [Co] and the discussions by Bellissard [Be2] and Kellendonk-Zois [KZ]. On the other hand, as pointed out by Bellissard and Scoppola in 1982 [BSc], a naive extension of gap labeling by means of the frequency module to discontinuous sampling functions may not work. Indeed, they exhibited an example where the potential arises by coding the rotation of the circle by $\alpha$ irrational with respect to two intervals whose lengths are irrational with respect to $\alpha$ and showed that the integrated density of states takes values not belonging to $\mathbb{Z}+\alpha \mathbb{Z}$.

In recent years, most of the literature on gap labeling has focused on aperiodic tilings and Delone sets in higher dimensions. While this is not immediately related to the subject of this paper, we do want to point out that a recent highlight was accomplished in three simultaneous and independent works by Bellissard-Benedetti-Gambaudo [BBG], Benameur-Oyono-Oyono [BO], and Kaminker-Putnam [KP]. They proved a version of the gap labeling theorem for aperiodic, repetitive tilings or Delone sets of finite local complexity.

\subsection{Cocycle dynamics}

It is well established that spectral properties of one-dimensional Schrödinger operators are intimately connected to a study of the solutions to the time-independent Schrödinger equation. This equation in turn can be reformulated in terms of transfer matrices. Since the potential is generated by sampling along the orbits of $f$ and the dependence of the transfer matrices on the potential is local, it turns out that these matrices have a so-called cocycle structure. In particular, the dynamics of these $\operatorname{SL}(2, \mathbb{R})$-valued cocycles describe the behavior of solutions and hence are closely related to spectral information. We will make this connection more explicit in the next subsection. Here, we discuss basic notions related to $\operatorname{SL}(2, \mathbb{R})$-valued cocycles and present a relative of Theorem 1 in this context.

Given a continuous map $A: X \rightarrow \operatorname{SL}(2, \mathbb{R})$, we consider the skew-product $X \times$ $\mathrm{SL}(2, \mathbb{R}) \rightarrow X \times \mathrm{SL}(2, \mathbb{R})$ given by $(x, g) \mapsto(f(x), A(x) \cdot g)$. This map is called the cocycle $(f, A){ }^{6}$ For $n \in \mathbb{Z}, A^{n}$ is defined by $(f, A)^{n}=\left(f^{n}, A^{n}\right)$. The Lyapunov exponent of the cocycle is the non-negative number

$$
L(A)=\lim _{n \rightarrow \infty} \frac{1}{n} \log \left\|A^{n}(x)\right\| .
$$

(The limit exists for $\mu$-almost every $x$.)

\footnotetext{
6 Since $f$ is fixed, we sometimes call $A$ a cocycle.
} 
We say that a cocycle $(f, A)$ is uniformly hyperbolic if there exist constants $c>0$ and $\lambda>1$ such that $\left\|A^{n}(x)\right\|>c \lambda^{n}$ for every $x \in X$ and $n>0 .{ }^{7}$ This is equivalent to the usual hyperbolic splitting condition: see [Y]. Thus uniform hyperbolicity is an open condition in $C(X, \operatorname{SL}(2, \mathbb{R}))$. Denote

$$
\mathcal{U H}=\{A \in C(X, \operatorname{SL}(2, \mathbb{R})):(f, A) \text { is uniformly hyperbolic }\} .
$$

We say that two cocycles $(f, A)$ and $(f, \tilde{A})$ are conjugate if there exists a conjugacy $B \in C(X, \operatorname{SL}(2, \mathbb{R}))$ such that $\tilde{A}(x)=B(f(x)) A(x) B(x)^{-1}$. Uniform hyperbolicity is invariant under conjugation.

Next suppose $A \in C(X, \operatorname{SL}(2, \mathbb{R}))$ is homotopic to a constant. Then one can define a homeomorphism $F: X \times \mathbb{R} \rightarrow X \times \mathbb{R}$ so that $F(x, t)=\left(f(x), F_{2}(x, t)\right)$ and $e^{2 \pi i F_{2}(x, t)}$ is a positive real multiple of $A(x) \cdot e^{2 \pi i t}$, with the usual identification of $\mathbb{C}$ with $\mathbb{R}^{2}$. We call $F_{2}(x, t)$ a lift of $A$. The lift is not uniquely defined, since for any continuous function $\phi: X \rightarrow \mathbb{Z},(x, t) \mapsto F_{2}(x, t)+\phi(x)$ is also a lift, but this is the only possible ambiguity.

Write $F_{2}^{n}(x, t)$ for the second component of $F^{n}(x, t)$. Then the limit

$$
\rho_{f, A}=\lim _{n \rightarrow \infty} \frac{F_{2}^{n}(x, t)-t}{n}
$$

exists uniformly and is independent of $(x, t)$, it is called the fibered rotation number of the cocycle $(f, A)$; compare $[H]$. Due to the ambiguity in the choice of $F_{2}, \rho$ is only defined up to addition of an element of the group $G^{\prime}=\left\{\int_{X} \phi d \mu: \phi \in C(X, \mathbb{Z})\right\}$. Clearly, $\mathbb{Z} \subset G^{\prime} \subset G$. Moreover, if $X$ is connected, then $G^{\prime}=\mathbb{Z}$, while if it is totally disconnected, then $G^{\prime}=G$.

We will be interested in the variation of the fibered rotation number. For any map $A_{0}: X \rightarrow \operatorname{SL}(2, \mathbb{R})$ that is homotopic to a constant, there is a neighborhood $\mathcal{V}$ of it in $C(X, \operatorname{SL}(2, \mathbb{R}))$ such that for each $A \in \mathcal{V}$, we can continuously select a lift $F_{A}: X \times \mathbb{R} \rightarrow$ $X \times \mathbb{R}$. Using those lifts, we define a map $A \in \mathcal{V} \mapsto \rho_{f, A} \in \mathbb{R}$, which is in fact continuous (see $[H, \S 5.7])$, and is called a continuous determination of the fibered rotation number.

It turns out that (see $[\mathrm{J}]$ )

$$
A \in \mathcal{U H} \Rightarrow 2 \rho_{f, A} \in G
$$

We will establish the following variant of Theorem 1 for $\operatorname{SL}(2, \mathbb{R})$-cocycles:

Theorem 3 (Accessibility by uniformly hyperbolic cocycles). Suppose $A \in$ $C(X, \operatorname{SL}(2, \mathbb{R}))$ is homotopic to a constant and obeys $2 \rho_{f, A} \in G$. Then there exists a continuous path $A_{t} \in C(X, \operatorname{SL}(2, \mathbb{R})), 0 \leq t \leq 1$, with $A_{0}=A$ and such that $A_{t} \in \mathcal{U H}$ for every $t>0$. (In particular, $\rho_{f, A_{t}}$ is independent of $t$.)

In fact, as we will see in the next subsection, Theorem 1 may be derived from Theorem 3 .

\footnotetext{
7 If $A$ is a real $2 \times 2$ matrix then $\|A\|=\sup _{\|v\| \neq 0}\|A(v)\| /\|v\|$, where $\|v\|$ is the Euclidean norm of $v \in \mathbb{R}^{2}$.
} 


\subsection{Schrödinger operators and relation to dynamics}

Let us now review in more detail the basic objects introduced at the beginning and their relation to the dynamics of cocycles.

Consider the operator $H=H_{f, v, x}$ given by (1). Since $f$ is minimal and $v$ is continuous, the $x$-independence of the spectrum $\Sigma_{f, v}$ of $H$ can be obtained from strong operator convergence modulo conjugation with shifts. This common spectrum has a convenient description in terms of the integrated density of states, which is defined as follows. There is a probability measure $d N_{f, v}$ that obeys

$$
\int\left\langle\delta_{0}, g(H) \delta_{0}\right\rangle d \mu(x)=\int g(E) d N_{f, v}(E)
$$

for every bounded, measurable function $g$. Indeed, by the spectral theorem, $d N_{f, v}$ is given by the $\mu$-average of the $x$-dependent spectral measures associated with $H$ and $\delta_{0}$. The distribution function of $d N_{f, v}$ is called the integrated density of states and denoted by $N_{f, v}$. By construction, it is a non-decreasing function from $\mathbb{R}$ onto $[0,1]$, which is constant in every connected component of $\mathbb{R} \backslash \Sigma_{f, v}$. Moreover, it is not hard to show that it is continuous and strictly increasing on $\Sigma_{f, v}$. That is, the measure $d N_{f, v}$ is non-atomic and its topological support is equal to $\Sigma_{f, v}$. There is an alternative way to define $N_{f, v}(E)$ by restricting $H$ to finite intervals, counting the eigenvalues below $E$, and taking a thermodynamic limit; compare [AS2]. A third way is explained below.

Spectral properties of general Schrödinger operators of the form (1) are most conveniently studied in terms of the solutions to the one-parameter family of difference equations

$$
u(n+1)+u(n-1)+V(n) u(n)=E u(n),
$$

where the energy $E$ belongs to $\mathbb{R}$. Equivalently,

$$
\left(\begin{array}{c}
u(n+1) \\
u(n)
\end{array}\right)=\left(\begin{array}{cc}
E-V(n) & -1 \\
1 & 0
\end{array}\right)\left(\begin{array}{c}
u(n) \\
u(n-1)
\end{array}\right) .
$$

Since in our situation, we have $V(n)=v\left(f^{n} x\right)$, we may consider the cocycle $\left(f, A_{E, v}\right)$, where

$$
A_{E, v}(x)=\left(\begin{array}{cc}
E-v(x) & -1 \\
1 & 0
\end{array}\right)
$$

and observe that $u$ solves (3) if and only if

$$
\left(\begin{array}{c}
u(n) \\
u(n-1)
\end{array}\right)=A_{E, v}^{n}\left(\begin{array}{c}
u(0) \\
u(-1) .
\end{array}\right) .
$$

Consequently, $(f, A)$ is called a Schrödinger cocycle if $A$ takes its values in the set

$$
\mathcal{S}=\left\{\left(\begin{array}{cc}
t & -1 \\
1 & 0
\end{array}\right): t \in \mathbb{R}\right\} \subset \operatorname{SL}(2, \mathbb{R}) .
$$


One of the fundamental results linking the spectral theory of $H$ and the dynamics of the family of Schrödinger cocycles $\left(f, A_{E, v}\right)$ is that $\Sigma_{f, v}$ consists of those energies $E$ for which $\left(f, A_{E, v}\right)$ is not uniformly hyperbolic:

$$
\mathbb{R} \backslash \Sigma_{f, v}=\left\{E \in \mathbb{R}:\left(f, A_{E, v}\right) \text { is uniformly hyperbolic }\right\} ;
$$

see Johnson [J].

The maps $A_{E, v}$ are homotopic to a constant and hence the cocycle $\left(f, A_{E, v}\right)$ has an associated rotation number which we denote by $\rho_{f, v}(E)$. For Schrödinger cocycles, there is a canonical way to remove the ambiguity in the definition of $\rho,{ }^{8}$ so that it can be interpreted as an element of $[0,1 / 2]$ (and not of $\mathbb{R} / G^{\prime}$ as in the general case). There is a simple relation between $N_{f, v}$ and $\rho_{f, v}$ which follows from Sturm oscillation theory:

$$
N_{f, v}(E)=1-2 \rho_{f, v}(E) .
$$

It follows from (2), (5), and (6) that the gaps of $\Sigma_{f, v}$ (i.e., the bounded connected components of $\left.\mathbb{R} \backslash \Sigma_{f, v}\right)$ may be labeled by elements of $\mathcal{L}=G \cap(0,1)$ so that the label of a gap corresponds to the constant value $N_{f, v}$ takes on it.

To deduce Theorem 1 from Theorem 3, we use the following result, which is an improved version of [ABD, Lemma 9] and is proved in Appendix D. (Recall that $\mathcal{S} \subset$ $\operatorname{SL}(2, \mathbb{R})$ indicates the set of Schrödinger matrices, as in (4).)

Lemma 1.3 (Projection Lemma). Let $f: X \rightarrow X$ be a minimal homeomorphism of a compact metric space with at least four points, and let $A \in C^{0}(X, \mathcal{S})$. Then there exist a neighborhood $\mathcal{W} \subset C^{0}(X, \operatorname{SL}(2, \mathbb{R}))$ of $A$ and continuous maps

$$
\Phi=\Phi_{A}: \mathcal{W} \rightarrow C^{0}(X, \mathcal{S}) \quad \text { and } \quad \Psi=\Psi_{A}: \mathcal{W} \rightarrow C^{0}(X, \operatorname{SL}(2, \mathbb{R}))
$$

satisfying

$$
\begin{gathered}
\Psi(B)(f(x)) \cdot B(x) \cdot[\Psi(B)(x)]^{-1}=\Phi(B)(x), \\
\Phi(A)=A \quad \text { and } \quad \Psi(A)=\mathrm{id} .
\end{gathered}
$$

In particular, an $\operatorname{SL}(2, \mathbb{R})$-valued perturbation of a Schrödinger cocycle is conjugate to an $\mathcal{S}$-valued perturbation.

Proof of Theorem 1. Take a sampling function $v \in C(X, \mathbb{R})$ and a label $\ell \in \mathcal{L}=G \cap$ $(0,1)$. Assume the gap of $\Sigma_{f, v}$ of label $\ell$ is collapsed, that is, there is a unique $E_{0} \in \mathbb{R}$ such that $N_{f, v}\left(E_{0}\right)=\ell$. Let

$$
A(x)=\left(\begin{array}{cc}
E_{0}-v(x) & -1 \\
1 & 0
\end{array}\right) .
$$

By (6), $\rho(A)=(1-\ell) / 2$. So we can apply Theorem 3 and find a continuous family of cocycles $A_{t}, t \in[0,1]$, with $A_{0}=A$ and $A_{t} \in \mathcal{U H}$ for $t>0$.

\footnotetext{
8 Namely, one can uniquely choose the lift $F_{2}$ so that $F_{2}(x, 1 / 4)=1 / 2$ for every $x \in X$.
} 
Apply Lemma 1.3 and define $\tilde{A}_{t}=\Phi_{A}\left(A_{t}\right)$ for small $t$. Since $\tilde{A}_{t} \in C(X, \mathcal{S})$, there is a continuous path $v_{t} \in C(X, \mathbb{R})$ so that

$$
\tilde{A}_{t}(x)=\left(\begin{array}{cc}
E_{0}-v_{t}(x) & -1 \\
1 & 0
\end{array}\right) .
$$

For $t>0$, we have $\tilde{A}_{t} \in \mathcal{U H}$ and so, by (5), $E_{0} \notin \Sigma_{f, v_{t}}$. Thus $N_{f, v_{t}}\left(E_{0}\right)$, which equals $1-2 \rho\left(\tilde{A}_{t}\right)=\ell$, is a label of an open gap.

\section{Statement of further results for $\operatorname{SL}(2, \mathbb{R})$-cocycles}

This paper actually contains more refined results on cocycles, and Theorem 3 is obtained as a corollary of one of them, as we now explain.

A cocycle $(f, A)$ is called bounded if there exists $C>1$ such that $\left\|A^{n}(x)\right\| \leq C$ for every $x \in X$ and $n \in \mathbb{Z}$. It is known that a cocycle is bounded if and only if it is conjugate to a cocycle of rotations, that is, to $(f, \tilde{A})$ with $\tilde{A}: X \rightarrow \mathrm{SO}(2, \mathbb{R})$; see [Ca], [EJ], [Y].

Our next main result is:

Theorem 4 (Accessibility by bounded cocycles). If $A \in C(X, \operatorname{SL}(2, \mathbb{R})) \backslash \mathcal{U H}$, then there exists a continuous path $A_{t} \in C(X, \mathrm{SL}(2, \mathbb{R})), t \in[0,1]$, such that $A_{0}=A$ and $\left(f, A_{t}\right)$ is conjugate to an $\mathrm{SO}(2, \mathbb{R})$-valued cocycle for every $t \in(0,1]$. Moreover, the conjugacy can be chosen to depend continuously on $t \in(0,1]$.

In particular, bounded cocycles are dense in the complement of $\mathcal{U H}$. In fact, we are able to prove this fact directly and with weaker hypotheses on the dynamics:

Theorem 5 (Denseness of bounded cocycles). Assume that $f$ is a minimal homeomorphism of a compact metric space $X$ such that at least one of the following holds:

(a) $X$ is finite-dimensional;

(b) $f$ has at most countably many distinct ergodic invariant measures, and $f$ has a nonperiodic finite-dimensional factor.

Then the bounded cocycles form a dense subset of $C(X, \operatorname{SL}(2, \mathbb{R})) \backslash \mathcal{U H}$.

We now focus on the case of cocycles that are homotopic to a constant, which form a subset of $C(X, \operatorname{SL}(2, \mathbb{R}))$ that we indicate by $C_{0}(X, \operatorname{SL}(2, \mathbb{R}))$.

Given $A \in C_{0}(X, \operatorname{SL}(2, \mathbb{R}))$, fix a continuous determination $\rho$ of the fibered rotation number on a neighborhood of it. We say that the cocycle $A$ is locked if $\rho$ is constant on a neighborhood of $A$. We say that $A$ is semi-locked if it is not locked and it is a point of local extremum for the function $\rho$. Otherwise we say that $A$ is unlocked. This classification is obviously independent of the chosen determination of $\rho$.

In fact, a cocycle is locked if and only if it is uniformly hyperbolic, and any semilocked cocycle $A$ can be accessed by uniformly hyperbolic cocycles. The proof of these statements and some related facts may be found in Appendix C. (Similar results for "nonlinear cocycles" over rotations can be found in $[\mathrm{BJ}]$.) 
For unlocked cocycles, we can strengthen the conclusion of Theorem 4 considerably:

Theorem 6 (Accessibility by cocycles conjugate to a rotation). Let $A \in C_{0}(X, \operatorname{SL}(2, \mathbb{R}))$ be an unlocked cocycle. Let $\alpha \in \mathbb{R}$ be such that $\alpha=\rho_{f, A} \bmod G$. Then there exist continuous paths $A_{t} \in C(X, \operatorname{SL}(2, \mathbb{R})), t \in[0,1]$, and $B_{t} \in C(X, \operatorname{SL}(2, \mathbb{R})), t \in(0,1]$, such that $A_{0}=A$ and $B_{t}(f(x))^{-1} A_{t}(x) B_{t}(x)=R_{2 \pi \alpha}$ for $t \in(0,1]$.

The assumption that the cocycle be unlocked is necessary: see Remark 10.6.

It is useful to consider also a weaker notion of conjugacy: We say that two cocycles $(f, A)$ and $(f, \tilde{A})$ are projectively conjugate if there exists a projective conjugacy $B \in C(X, \operatorname{PSL}(2, \mathbb{R}))$ such that $\tilde{A}(x)=B(f(x)) A(x) B(x)^{-1}$ modulo sign. The corresponding version of Theorem 6 is:

Theorem 7 (Accessibility by cocycles projectively conjugate to a rotation). Let $A \in$ $C_{0}(X, \operatorname{SL}(2, \mathbb{R}))$ be an unlocked cocycle. Let $\alpha \in \mathbb{R}$ be such that $2 \alpha=2 \rho_{f, A} \bmod G$. Then there exist continuous paths $A_{t} \in C(X, \operatorname{SL}(2, \mathbb{R})), t \in[0,1]$, and $B_{t} \in$ $C(X, \operatorname{PSL}(2, \mathbb{R})), t \in(0,1]$, such that $A_{0}=A$ and $B_{t}(f(x))^{-1} A_{t}(x) B_{t}(x)=R_{2 \pi \alpha}$ (modulo sign) for $t \in(0,1]$.

This result easily yields Theorem 3:

Proof of Theorem 3. Suppose $A \in C(X, \mathrm{SL}(2, \mathbb{R}))$ is homotopic to a constant with $2 \rho_{f, A} \in G$; we want to access $A$ by a path $A_{t}$ in $\mathcal{U H}$. As mentioned above, if $A$ is locked, then $A$ is in $\mathcal{U H}$ and thus we can take a constant path. If $A$ is semi-locked, then a path of the form $A_{t}=R_{\varepsilon t} A$ (for some small $\varepsilon \neq 0$ ) works; see Proposition C.1. Thus assume that $A$ is unlocked.

Apply Theorem 7 with $\alpha=0$ and find continuous paths $A_{t} \in C(X, \operatorname{SL}(2, \mathbb{R}))$, $t \in[0,1]$, and $B_{t} \in C(X, \operatorname{PSL}(2, \mathbb{R})), t \in(0,1]$, such that $A_{0}=A$ and $A_{t}(x)=$ $B_{t}(f(x)) B_{t}(x)^{-1}$ (modulo sign) for $t \in(0,1]$. Let $\tau:(0,1] \rightarrow(1, \infty)$ be a continuous function. Take $\tilde{A}_{t} \in C(X, \operatorname{SL}(2, \mathbb{R}))$ such that

$$
\tilde{A}_{t}(x)=B_{t}(f(x))\left(\begin{array}{cc}
\tau(t) & 0 \\
0 & 1 / \tau(t)
\end{array}\right) B_{t}(x)^{-1} \quad \text { (modulo sign). }
$$

Choosing a function $\tau(t)$ that goes sufficiently fast to 1 as $t \rightarrow 0$, we have $\lim _{t \rightarrow 0} \tilde{A}_{t}(x)$ $=A(x)$. This path has the desired properties.

As for uniformly hyperbolic cocycles, they cannot, in general, be approximated by cocycles that are conjugate to a constant (see Remark 3 in [ABD]). But for projective conjugacy there is no obstruction; indeed we show:

Theorem 8 (Accessibility by cocycles projectively conjugate to a hyperbolic matrix). Let $A \in \mathcal{U H}$, and let $D \in \mathrm{SL}(2, \mathbb{R})$ be such that $L(A)=L(D)$. Then there exist continuous paths $A_{t} \in C(X, \operatorname{SL}(2, \mathbb{R})), t \in[0,1]$, and $B_{t} \in C(X, \operatorname{PSL}(2, \mathbb{R})), t \in(0,1]$, such that $A_{0}=A$ and $B_{t}(f(x)) A_{t}(x) B_{t}(x)^{-1}=D($ in $\operatorname{PSL}(2, \mathbb{R}))$ for $t \in(0,1]$. 
Remark 2.1. Cocycles that are projectively conjugate to a constant are called reducible. Theorems 7 and 8 (together with the fact that the set of semi-locked cocycles has empty interior) imply that reducibility is dense in $C_{0}(X, \operatorname{SL}(2, \mathbb{R}))$. As for uniform hyperbolicity, it is dense in $C_{0}(X, \operatorname{SL}(2, \mathbb{R}))$ if and only if $G$ is a non-discrete subgroup of $\mathbb{R}$, by Theorem 3 (and the basic fact (2)). This statement cannot be significantly improved; compare Theorem 9 in Appendix B.

More generally, as in [ABD], we can let Ruth be the set of all cocycles that are reducible up to homotopy (or, equivalently, are projectively conjugate to a cocycle $C_{0}(X, \mathrm{SL}(2, \mathbb{R}))$; see Lemma 8 in $\left.[\mathrm{ABD}]\right)$. Any uniformly hyperbolic cocycle is reducible up to homotopy, and in [ABD] it is shown that reducible uniformly hyperbolic cocycles are dense in Ruth in the particular case where $f$ is a generalized skew-product. Our results imply that in our more general context, reducibility is still dense in Ruth, and uniformly hyperbolic cocycles are dense in Ruth if and only if $G$ is non-discrete.

Remark 2.2. We also notice the following consequence of our methods: any continuous cocycle $A$ that is not uniformly hyperbolic admits arbitrarily small neighborhoods in $C(X, \operatorname{SL}(2, \mathbb{R})) \backslash \mathcal{U H}$ that are path connected (and even have trivial homotopy groups in all dimensions). See the comment after Proposition 9.2.

\section{Outline of the paper and discussion of the methods}

Most of the paper is dedicated to building up the tools that will be involved in the proof of cousin Theorems 6 and 7, with the other results being obtained on the side. These tools can be split into two classes of results which produce families of cocycles with certain features. The input in either case is always some initial family of cocycles, but while the first kind of result is only concerned with outputting a suitable perturbation of the family, the second is primarily concerned with the extension of the parameter space.

The perturbation arguments are developed in Sections 4 to 7: they refine the ideas introduced in [ABD] to deal with individual cocycles over generalized skew-shifts, both to address a more general class of dynamical systems and to allow us to work in the context of parameterized families. Since many problems about cocycles can be rephrased in terms of the existence of invariant sections for an associated skew-product (for instance, conjugacy to rotations is equivalent to the existence of an invariant section for the associated skew-product arising from the disk action of $\operatorname{SL}(2, \mathbb{R})$ ), we first (Section 4) discuss in a more abstract setting the problem of existence of almost invariant sections, satisfying the invariance condition except in some specific set. The actual estimates for the section depend on the recurrence properties of the set and the existence of sets with good recurrence properties is the topic of Section 5. Those results are used in Section 6 to show that a family of SL $(2, \mathbb{R})$-cocycles with small Lyapunov exponents can be perturbed to become continuously conjugate to a family of cocycles of rotations (Proposition 6.3); hence Theorem 5 follows as a particular case. A similar result (Lemma 7.1), regarding the cohomological equation (which plays a role when conjugating a cocycle of rotations to a constant), is obtained in Section 7, and permits us to establish Theorem 8. 
The extension arguments are developed in Sections 8 and 9: the questions considered are rather novel, and their proofs need the introduction of several ideas. In Section 8 we show that a family of cocycles with zero Lyapunov exponent, defined over the boundary of a cube, can be extended to a family of cocycles with small Lyapunov exponents, defined over the whole cube, provided there is no topological obstruction (Lemma 8.1). The proof is technically quite involved, jumping from the continuous to the measurable category and back, and is mostly independent of the rest of the paper (which never departs from the continuous category): it is also the only place where ergodic theory plays a significant role. In Section 9, Lemma 8.1 is used to bootstrap a considerably more refined result (Proposition 9.2): under the weaker condition that the boundary values are not uniformly hyperbolic, one gets the stronger conclusion that the extended family can be continuously conjugated to cocycles of rotations in the interior of the cube. In particular, we obtain Theorem 4.

In Section 10, everything is put together in the proof of Theorems 6 and 7. We first show (Lemma 10.3) that given $A$ unlocked, it is possible to construct a two-parameter family $A_{s, t},(s, t) \in[0,1]^{2}$ with $A_{0,0}=A$, such that $\rho\left(A_{0, s}\right)<\rho(A)<\rho\left(A_{s, 0}\right)$, and which is continuously conjugate to rotations (except possibly at $(0,0)$ ). This almost implies that $A$ is accessible from the interior of the square $[0,1]^{2}$ through a path with constant rotation number. The actual accessibility of $A$ by cocycles conjugate to a fixed rotation is obtained with a little wiggling involving in particular the solution of the cohomological equation.

The following diagram gives the logical dependence between the sections:

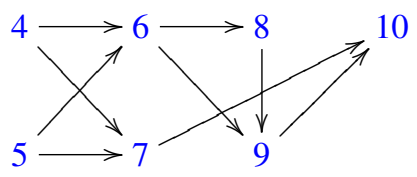

The paper also contains five appendices. The first contains complementary results and examples concerning connectedness of some sets of cocycles. The second shows that complete gap labeling, and even just the Cantor structure of the spectrum, ceases to be generic when the assumption of unique ergodicity is dropped. The third presents some facts on locked / semi-locked / unlocked cocycles. The fourth proves the projection lemma that was used to realize $\operatorname{SL}(2, \mathbb{R})$ perturbations by Schrödinger matrices. Finally, in the last appendix we show how Fayad's results can be applied to obtain a mixing strictly ergodic example with a dense set of labels.

\section{Construction of almost invariant sections}

In this section and in the next section, $f: X \rightarrow X$ is an arbitrary homeomorphism of a compact metric space. In fact, even if we are ultimately interested in minimal dynamical systems, in order to obtain "parameterized" results in the later sections, we will apply the results obtained here to maps of the form $\hat{f}(x, t)=(f(x), t)$ (the second coordinate representing the parameter). 
Let $Y$ be a topological space and let $F: X \times Y \rightarrow X \times Y$ be an invertible skew-product over $f$, that is, a homeomorphism of the form $(x, y) \mapsto\left(f(x), F_{x}(y)\right)$. An invariant section for $F$ is a continuous map $y: X \rightarrow Y$ such that $F_{x}(y(x))=y(f(x))$. Throughout the paper, a common theme will be the construction of almost invariant sections, which satisfy $F_{x}(y(x))=y(f(x))$ in much of $X$. We define the support of an almost invariant section as the set of all $x$ such that $F_{x}(y(x)) \neq y(f(x))$. We will also be interested in estimating "how large" such almost invariant sections are. In the abstract setting considered here, this largeness will be measured by a continuous function $M: Y \rightarrow[0, \infty)$.

We say that the pair $(Y, M)$ has the Tietze property if for any metric space $L$, every continuous function $y: K \rightarrow Y$ defined on a compact subset $K \subset L$ can be extended to a continuous map $y: L \rightarrow Y$ such that

$$
\sup _{x \in L} M(y(x))=\sup _{x \in K} M(y(x)) .
$$

The following are the examples of pairs $(Y, M)$ that we will use:

- $Y=\mathbb{R}$ and $M(y)=|y|$;

- $Y$ is the unit disk $\mathbb{D}$ and $M(y)=\mathrm{d}(y, 0)$, where $\mathrm{d}$ is the hyperbolic distance (see $\S 6.1$ ).

Using Tietze's Extension Theorem, we see that the Tietze property holds in these two examples.

We denote

$$
\|F\|_{M}=\sup _{(x, y) \in X \times Y}\left|M\left(F_{x}(y)\right)-M(y)\right| \in[0, \infty] .
$$

Notice that $\left\|\left|F^{-1}\left\|_{M}=\right\|\right| \mid F\right\|_{M}$.

To construct and estimate almost invariant sections whose support is contained in the interior of a compact set $K$, we will need only a few aspects of the recurrence of the dynamics to $K$, which we encode in the following notions. We say that $K \subset X$ is $n$-good if its first $n$ iterates are disjoint, that is, $f^{i}(K) \cap K=\emptyset$ for $1 \leq i \leq n-1$. Let us say that $K \subset X$ is $N$-spanning if the first $N$ iterates cover $X$, that is, $\bigcup_{i=0}^{N-1} f^{i}(K)=X$. We say that $K \subset X$ is $d$-mild if the orbit $\left\{f^{n} x: n \in \mathbb{Z}\right\}$ of each point in $X$ enters $\partial K$ at most $d$ times. The construction of sets with appropriate recurrence properties, under suitable dynamical assumptions, will be carried out in the next section.

The following abstract lemma is the main result of this section. (A simple particular case of it, where the dynamics fibers over an irrational rotation, corresponds to Lemma 2 from $[\mathrm{ABD}]$.)

Lemma 4.1. Let $f: X \rightarrow X$ be a homeomorphism of a compact metric space $X$, and let $K \subset X$ be a d-mild, $n$-good, $N$-spanning set. Let $\Lambda \subset X$ be a compact (possibly empty) $f$-invariant set. Let $(Y, M)$ be a pair with Tietze property, and let $F: X \times Y \rightarrow X \times Y$ be an invertible skew-product over $f$. Let $y_{0}: X \rightarrow Y$ be an almost invariant section supported outside $\Lambda$. Then there exists an almost invariant section $y: X \rightarrow Y$ supported in int $K$ with the following properties:

(a) y equals $y_{0}$ on $\Lambda$;

(b) $\sup _{x \in X} M(y(x)) \leq \sup _{x \in X} M\left(y_{0}(x)\right)+d \max _{j \in[n, N]}\left\|\left|F^{j}\right|\right\|_{M}$. 
Proof. We begin by using the set $K$ and the dynamics $f$ to decompose the space $X$.

For each $x \in X$, let

$$
\begin{gathered}
\ell^{+}(x)=\min \left\{j \geq 0: f^{j}(x) \in \text { int } K\right\}, \quad \ell^{-}(x)=\min \left\{j>0: f^{-j}(x) \in \text { int } K\right\}, \\
T(x)=\left\{j \in \mathbb{Z}:-\ell^{-}(x)<j<\ell^{+}(x)\right\}, \quad T_{B}(x)=\left\{j \in T(x): f^{j}(x) \in \partial K\right\} .
\end{gathered}
$$

Notice $T$ and $T_{B}$ are upper-semicontinuous. Define $N(x)=\# T_{B}(x)$ and $X^{i}=\{x \in X$ : $N(x) \geq d-i\}$. The sets $X^{i}$ are closed and

$$
X=X^{d} \supset X^{d-1} \supset \cdots \supset X^{0} \supset X^{-1}=\emptyset .
$$

Let also $Z^{i}=X^{i} \backslash X^{i-1}=\{x: N(x)=d-i\}$.

Claim. $\ell^{+}$is locally constant on the set $Z^{i}$.

Proof. We will show that $T_{B}$ and $T$ are locally constant on $Z^{i}$. Fix $x \in Z^{i}$ and let $y \in Z^{i}$ be very close to $x$. We have $T_{B}(y) \subset T_{B}(x)$. Since $y$ is also in $Z^{i}$, we must have equality. Now, if $j \in T(x)$, then either $f^{j}(x) \in \partial K$ or $f^{j}(x) \in X \backslash K$. In the former case, $f^{j}(y) \in \partial K$ (because $T_{B}(y)=T_{B}(x)$ ), and in the latter, $f^{j}(y)$ is also in $X \backslash K$ (because $y$ is close to $x$ ). Since $f^{\ell^{+}(x)}(y), f^{-\ell^{-}(x)}(y) \notin$ int $K$, we conclude that $T(y)=T(x)$.

Let $Z_{\ell}^{i}=\left\{x \in Z^{i}: \ell^{+}(x)=\ell\right\}$; by the claim this is a (relatively) open subset of $X^{i}$. Also notice the following facts:

$$
\begin{gathered}
X^{i}=X^{i-1} \sqcup Z_{0}^{i} \sqcup Z_{1}^{i} \sqcup \cdots \sqcup Z_{N}^{i}, \quad \overline{Z_{\ell}^{i}} \backslash Z_{\ell}^{i} \subset X^{i-1}, \\
Z_{0}^{i}=Z^{i} \cap \operatorname{int} K, \quad f\left(Z_{\ell}^{i}\right)=Z_{\ell-1}^{i} \text { if } \ell>0 .
\end{gathered}
$$

Now let $(Y, M), F, \Lambda, y_{0}$ be as in the assumptions of the lemma. We will describe a procedure to successively define $y$ on the set $\Lambda \cup\left(X^{0} \cap\right.$ int $\left.K\right)$, then on $\Lambda \cup X^{0}$, then on $\Lambda \cup X^{0} \cup\left(X^{1} \cap\right.$ int $\left.K\right)$, then on $\Lambda \cup X^{1}$, then on $\Lambda \cup X^{1} \cup\left(X^{2} \cap\right.$ int $\left.K\right), \ldots$, then on $\Lambda \cup X^{d-1} \cup\left(X^{d} \cap\right.$ int $\left.K\right)$, and finally on $\Lambda \cup X^{d}=X$.

We begin by defining $y$ on $\Lambda \cup\left(X^{0} \cap\right.$ int $\left.K\right)$ as equal to $y_{0}$.

Let $i \in\{0, \ldots, d\}$. Assume, by induction, that the map $y$ has already been continuously defined on the set $\Lambda \cup X^{i-1} \cup\left(X^{i} \cap\right.$ int $\left.K\right)$, and has the two properties below:

$$
\begin{gathered}
F(x, y(x))=(f(x), y(f(x))) \quad \text { for every } x \text { in the domain and not in int } K, \\
\sup _{x \in \Lambda \cup X^{i-1} \cup\left(X^{i} \cap \operatorname{int} K\right)} M(y(x)) \leq \sup _{x \in X} M\left(y_{0}(x)\right)+i A,
\end{gathered}
$$

where $A=\max _{j \in[n, N]}\left\|\left|F^{j}\right|\right\|_{M}$.

Now, for each $\ell=1, \ldots, N$, the map $y$ is already defined on $f^{\ell}\left(Z_{\ell}^{i}\right) \subset X^{i} \cap \operatorname{int} K$. Naturally, we define $y$ on $Z_{\ell}^{i}$ by

$$
y(x)=\left(F_{x}^{\ell}\right)^{-1}\left(y\left(f^{\ell}(x)\right)\right) .
$$

This defines $y$ on the set $\Lambda \cup X^{i}$ in such a way that (7) holds, and moreover

$$
\sup _{x \in \Lambda \cup X^{i}} M(y(x)) \leq \sup _{x \in \Lambda \cup X^{i-1} \cup\left(X^{i} \cap \operatorname{int} K\right)} M(y(x))+A .
$$


Let us check that the map $y$ so defined on $\Lambda \cup X^{i}$ is continuous. Since $\Lambda$ and $X_{i}$ are closed sets and $y\left|\Lambda=y_{0}\right| \Lambda$ is continuous, we only need to check that $y \mid X^{i}$ is continuous. Take a sequence $x_{j}$ in $X^{i}$ converging to some $x$ and let us show that $y\left(x_{j}\right) \rightarrow y(x)$. If $x \in Z^{i}$, convergence follows from the claim above and the continuity of $y \mid X^{i} \cap$ int $K$. Next assume $x \in X^{i-1}$. Since $X^{i}=X^{i-1} \sqcup Z_{0}^{i} \sqcup Z_{1}^{i} \sqcup \cdots \sqcup Z_{N}^{i}$, and $y \mid X^{i-1}$ is continuous, it suffices to consider the case where the sequence $x_{j}$ is contained in some $Z_{\ell}^{i}$. Then $f^{\ell}\left(x_{j}\right)$ and $f^{\ell}(x)$ all belong to $\left(X^{i} \cap \operatorname{int} K\right) \cup X^{i-1}$, where $y$ is continuous. So, applying $F^{-\ell}$, we obtain $y(x)=\lim y\left(x_{j}\right)$, as desired.

At this point, $y$ is continuously defined on $\Lambda \cup X^{i}$. If $i=d$, we are done. Otherwise, we use the Tietze property to extend continuously $y$ to $\Lambda \cup X^{i} \cup\left(X^{i+1} \cap\right.$ int $\left.K\right)$ in a way such that

$$
\sup _{x \in \Lambda \cup X^{i} \cup\left(X^{i+1} \cap \operatorname{int} K\right)} M(y(x))=\sup _{x \in \Lambda \cup X^{i}} M(y(x)) .
$$

Then the hypotheses (7) and (8) hold with $i+1$ in place of $i$; thus we can increment $i$ and continue the procedure.

\section{Construction of sets with controlled return times}

In the applications of Lemma 4.1, it would be bad if $N$ was much larger than $n$ (because then we would not get a useful estimate for the $M$-size of the almost invariant section). This issue is settled by means of the following result:

Proposition 5.1. Let $f: X \rightarrow X$ be a homeomorphism of a compact metric space that admits a non-periodic minimal finite-dimensional factor. Then there is $d \in \mathbb{N}$ such that for every $n \in \mathbb{N}$, there is a compact set $K \subset X$ that is $n$-good, $(d+2) n-1$-spanning, and d-mild.

We mention that in the case where $f$ fibers over an irrational rotation, the proposition can be proven (with $d=1$ ) by a simple explicit construction based on continued fractionssee Figure 1 from $[\mathrm{ABD}]$.

If $\tilde{f}: \tilde{X} \rightarrow \tilde{X}$ is a factor of $f: X \rightarrow X$ and $\tilde{K} \subset \tilde{X}$ is $d$-mild, $n$-good and $N$ spanning for $\tilde{f}$, then $\pi^{-1}(\tilde{K})$ is $d$-mild, $n$-good and $N$-spanning for $\tilde{f}$, where $\pi: X \rightarrow \tilde{X}$ is a continuous surjective map such that $\pi \circ f=\tilde{f} \circ \pi$. Thus it suffices to prove this statement for the minimal non-periodic finite-dimensional factor of $f$. For the remainder of this section, it will therefore be assumed that $X$ is finite-dimensional and infinite and that $f$ is minimal.

Lemma 5.2. There exists $d \in \mathbb{N}$ such that for every compact set $K \subset X$ and every neighborhood $U$ of $K$, there exists a compact neighborhood $K^{\prime} \subset U$ of $K$ that is $d$-mild.

Proof. This follows easily from Lemmas 4 and 5 in [AB].

Let now $d$ given by Lemma 5.2 be fixed.

Lemma 5.3. Let $K \subset X$ be an $n$-good, $N$-spanning compact set. Then there exists $a$ compact neighborhood $K^{\prime}$ of $K$ that is $n$-good, $N$-spanning and $d$-mild. 
Proof. If $K^{\prime}$ is a compact neighborhood of $K$ sufficiently close to $K$, then it is still $n$ good and $N$-spanning. So the result follows from Lemma 5.2.

Lemma 5.4. For every $n \in \mathbb{N}$, there exists an $n$-good, $(d+2) n-1$-spanning compact set $K \subset X$.

Proof. Let $n$ be fixed. Let us start with a compact set $K$ with non-empty interior whose first $n$ iterates are disjoint (a small compact neighborhood of any point will do). By minimality, $K$ is $n$-good and $N$-spanning for some $N$. Let us show that if $N \geq(d+2) n$, then there exists an enlargement of $K$ that is $n$-good and $N-1$-spanning.

Assume $K$ is not $N-1$-spanning. Let $K^{\prime}$ be a compact neighborhood of $K$ that is $n$-good, $N$-spanning and $d$-mild, and also not $N-1$-spanning. Let $K_{*}$, resp. $K_{*}^{\prime}$, be the set of $x$ in $K$, resp. $K^{\prime}$, such that for each $i$ with $1 \leq i<N$, the point $f^{i}(x)$ does not belong to $K$, resp. $K^{\prime}$. Let $Y$ be the non-empty set $\overline{K_{*}^{\prime}}$. Notice that $Y$ is contained in $K_{*}$ and thus is $N$-good. To simplify notation, replace $K$ with $K^{\prime}$.

If $x \in Y$ and $f^{j}(x) \in K$ with $1 \leq j \leq N-1$, then $f^{j}(x) \in \partial K$. For $x \in Y$, let $J(x)$ be the set of all $j$ with $1 \leq j \leq N-1$ and $f^{j}(x) \notin K$. Let $I(x)$ be the set of all $i$ with $1 \leq i \leq d+1$ such that $\{i n, i n+1, \ldots,(i+1) n-1\} \subset J(x)$. Since $K$ is $d$-mild, $\{1, \ldots, N-1\} \backslash J(x)$ has at most $d$ elements and $I(x) \neq \emptyset$ for every $x \in Y$.

If for each $x \in Y$ we choose a non-empty subset $\tilde{I}(x)$ of $I(x)$, then the formula

$$
K^{\prime}=K \cup \bigcup_{x \in Y} \bigcup_{i \in I_{\varepsilon}(x)} f^{i n}(x)
$$

defines an $n$-good and $N-1$-spanning set. For $K^{\prime}$ to be compact, we need to guarantee that

$$
\text { for any } i \text {, the set }\{x \in Y: \tilde{I}(x) \ni i\} \text { is closed. }
$$

For $x \in Y$ and $\varepsilon>0$, let $B(x, \varepsilon)=\{y \in Y: d(y, x)<\varepsilon\}$. Let $J_{\varepsilon}(x)=$ $\bigcap_{y \in B(x, \varepsilon)} J(y)$ and $I_{\varepsilon}(x)=\bigcap_{y \in B(x, \varepsilon)} I(y)$. For any $x \in Y$, there is $\varepsilon(x)>0$ such that $J_{\varepsilon(x)}(x)=J(x)$, and hence $I_{\varepsilon(x)}(x)=I(x)$. Since $Y$ is compact, it can be covered by finitely many balls $B\left(x_{k}, \varepsilon\left(x_{k}\right)\right)$. Let $\varepsilon$ be a Lebesgue number for this cover. Then, for each $x \in Y$, there exists some $k$ such that $B(x, \varepsilon) \subset B\left(x_{k}, \varepsilon\left(x_{k}\right)\right)$ and therefore

$$
I_{\varepsilon}(x)=\bigcap_{y \in B(x, \varepsilon)} I(y) \supset \bigcap_{y \in B\left(x_{k}, \varepsilon\left(x_{k}\right)\right)} I(y)=I_{\varepsilon\left(x_{k}\right)}\left(x_{k}\right)=I\left(x_{k}\right) \neq \emptyset .
$$

Defining $\tilde{I}(x)=I_{\varepsilon}(x)$, it is easy to see that (9) is satisfied. Thus there exist an $n$-good and $N-1$-spanning compact set, as we wanted to show.

Remark 5.5. Motivated by this result, we pose the following question: If $f$ is a homeomorphism of a compact space (possibly of infinite dimension) without periodic points, is it true that for every $n \in \mathbb{N}$, there exists a compact set whose first $n$ iterates are disjoint and such that finitely many iterates cover the whole space? A related result is Theorem 3.1 in $[\mathrm{BC}]$.

Proposition 5.1 follows readily from the previous lemmas. 


\section{From slow growth to invariant sections for the disk action}

\subsection{The disk action and the adjustment lemma}

The group $\operatorname{SL}(2, \mathbb{R})$ acts on the upper half-plane $\mathbb{H}=\{w \in \mathbb{C}: \operatorname{Im} z>0\}$ as follows:

$$
A=\left(\begin{array}{ll}
a & b \\
c & d
\end{array}\right) \in \operatorname{SL}(2, \mathbb{R}) \Rightarrow A \cdot w=\frac{a w+b}{c w+d}
$$

(In fact, the action factors through $\operatorname{PSL}(2, \mathbb{R})$.) We fix the following conformal equivalence between $\mathbb{H}$ and the disk $\mathbb{D}=\{z \in \mathbb{C}:|z|<1\}$ :

$$
w=\frac{-i z-i}{z-1} \in \mathbb{H} \leftrightarrow z=\frac{w-i}{w+i} \in \mathbb{D} .
$$

Conjugating through this equivalence, we get an action of $\operatorname{SL}(2, \mathbb{R})$ on $\mathbb{D}$, which we also denote as $(A, z) \mapsto A \cdot z$.

The disk is endowed with the Riemannian metric

$$
v \in T_{z} \mathbb{D} \Rightarrow\|v\|_{z}=\frac{2|v|}{1-|z|^{2}} .
$$

Let $\mathrm{d}(\cdot, \cdot)$ denote the induced distance function. The group $\operatorname{SL}(2, \mathbb{R})$ acts on $\mathbb{D}$ by isometries.

It can be shown that

$$
\|A\|=e^{\mathrm{d}(A \cdot 0,0) / 2} \quad \text { for all } A \in \mathrm{SL}(2, \mathbb{R}) .
$$

In particular, $A \in \mathrm{SO}(2, \mathbb{R})$ iff $A \cdot 0=0$.

Let us recall Lemmas 5 and 6 from $[\mathrm{ABD}]$ :

Lemma 6.1. There exists a continuous map $\Phi: \mathbb{D} \times \mathbb{D} \rightarrow \operatorname{SL}(2, \mathbb{R})$ such that $\Phi\left(p_{1}, p_{2}\right)$. $p_{1}=p_{2}$ and $\left\|\Phi\left(p_{1}, p_{2}\right)-\mathrm{Id}\right\| \leq e^{\mathrm{d}\left(p_{1}, p_{2}\right)}-1$.

Lemma 6.2 (Disk Adjustment). For every $n \geq 1$, there exists a continuous map $\Psi_{n}$ : $\operatorname{SL}(2, \mathbb{R})^{n} \times \mathbb{D}^{2} \rightarrow \operatorname{SL}(2, \mathbb{R})^{n}$ such that if $\Psi_{n}\left(\bar{A}_{1}, \ldots, A_{n}, p, q\right)=\left(\tilde{A}_{1}, \ldots, \tilde{A}_{n}\right)$, then

(a) $\tilde{A}_{n} \cdots \tilde{A}_{1} \cdot p=q$;

(b) $\left\|\tilde{A}_{i} A_{i}^{-1}-\mathrm{Id}\right\| \leq \exp \left(\frac{1}{2 n} \mathrm{~d}\left(A_{n} \cdots A_{1} \cdot p, q\right)\right)-1$ for $1 \leq i \leq n$.

\subsection{Construction of an invariant section}

Let $f: X \rightarrow X$ be a homeomorphism of a compact metric space. If $A \in C(X, \operatorname{SL}(2, \mathbb{R}))$, the cocycle $(f, A)$, as defined in the introduction, is a skew-product on $X \times \operatorname{SL}(2, \mathbb{R})$. The disk action of $\operatorname{SL}(2, \mathbb{R})$ allows us to consider also another skew-product denoted by $F_{f, A}^{\mathbb{D}}: X \times \mathbb{D} \rightarrow X \times \mathbb{D}$ and given by $F_{f, A}^{\mathbb{D}}(x, z)=(x, A(x) \cdot z)$. The existence of an invariant section for $F_{f, A}^{\mathbb{D}}$ is easily seen to be equivalent to $(f, A)$ being conjugate to a cocycle of rotations. Indeed, if $\Phi$ is given by Lemma 6.1 and $B(x)=\Phi(z(x), 0)$, then $B(f(x)) A(x) B(x)^{-1}$ is a rotation for every $x$.

If $A \in C(X, \operatorname{SL}(2, \mathbb{R})$ ) (or, more, generally, $A(x)$ is a $2 \times 2$ real matrix depending continuously on $x \in X$ ), we denote $\|A\|_{\infty}=\sup _{x \in X}\|A(x)\|$. 
Proposition 6.3. Let $f: X \rightarrow X$ be a homeomorphism of a compact metric space that admits a minimal non-periodic finite-dimensional factor, and let $\Lambda \subset X$ be a (possibly empty) compact invariant set. For every $C, \varepsilon>0$, there exists $\gamma>0$ such that the following holds. Suppose that A $: X \rightarrow \mathrm{SL}(2, \mathbb{R})$ is continuous,

$$
\|A\|_{\infty}<C \quad \text { and } \quad \lim _{n \rightarrow \infty} \sup _{x \in X} \frac{1}{n} \log \left\|A^{n}(x)\right\|<\gamma,
$$

and that $F_{f|\Lambda, A| \Lambda}^{\mathbb{D}}$ admits an invariant section $z$. Then $A \mid \Lambda$ extends to a continuous map $\tilde{A}: X \rightarrow \operatorname{SL}(2, \mathbb{R})$ such that $\|\tilde{A}-A\|_{\infty}<\varepsilon$ and $z$ extends to an invariant section $\tilde{z}$ for $F_{f, \tilde{A}}^{\mathbb{D}}$.

Proof. Let $d$ be given by Proposition 5.1. Let $\gamma>0$ be such that $e^{[1+2 d(d+1)] \gamma}<1+\varepsilon / C$. Let $n_{0}$ be such that $n \geq n_{0}$ implies $\left\|A^{n}(x)\right\| \leq e^{n \gamma}$ for every $x \in X$.

Let $M: \mathbb{D} \rightarrow[0, \infty)$ be the hyperbolic distance to 0 . Fix some extension of $z$ to an element of $C(X, \mathbb{D})$ and set $C_{1}=\sup _{x \in X} M(z(x))$. Choose $n \in \mathbb{N}$ so that $n>$ $\max \left\{n_{0}, \gamma^{-1} \log C, C_{1} / \gamma\right\}$. Let $K$ be the corresponding $d$-mild, $n$-good, $N$-spanning set given by Proposition 5.1, where $N=(d+2) n-1$.

We have

$$
\begin{aligned}
\left\|\left(F_{f, A}^{\mathbb{D}}\right)^{j}\right\| \|_{M} & =\sup _{x \in X} \sup _{w \in \mathbb{D}}\left|\mathrm{d}\left(A^{j}(x) \cdot w, 0\right)-\mathrm{d}(w, 0)\right| & & \text { (by definition) } \\
& \leq \sup _{x \in X}\left|\mathrm{~d}\left(A^{j}(x) \cdot 0,0\right)\right| & & \text { (since dis } \operatorname{SL}(2, \mathbb{R}) \text {-invariant) } \\
& \leq 2 \sup _{x \in X} \log \left\|A^{j}(x)\right\| & & \text { (by (10)) } \\
& \leq 2 j \gamma, & & \text { provided } j \geq n>n_{0} .
\end{aligned}
$$

Applying Lemma 4.1 we obtain an almost invariant section $\hat{z}$ for $F_{f, A}^{\mathbb{D}}$, which is supported in int $K$ and satisfies

$$
\sup _{x \in X} M(\hat{z}(x)) \leq \sup _{x \in X} M(z(x))+d \max _{n \leq j \leq N}\left\|\left(F_{f, A}^{\mathbb{D}}\right)^{j}\right\|_{M} .
$$

Thus we obtain $M(\hat{z}(x)) \leq C_{1}+2 d N \gamma$ for every $x \in X$.

Let $\Psi_{n}$ be given by Lemma 6.2 and put

$$
\begin{aligned}
\left(\tilde{A}(x), \tilde{A}(f(x)), \ldots, \tilde{A}\left(f^{n-1}(x)\right)\right) \\
\quad=\Psi_{n}\left(A(x), A(f(x)), \ldots, A\left(f^{n-1}(x)\right), \hat{z}(x), \hat{z}\left(f^{n}(x)\right)\right)
\end{aligned}
$$

for each $x \in K$. This defines $\tilde{A}$ on $\bigsqcup_{m=0}^{n-1} f^{m}(K)$. We let $\tilde{A}=A$ on the rest of $X$. Clearly $\tilde{A}$ is a continuous extension of $A \mid \Lambda$.

For each $x \in K$, and $1 \leq m \leq n-1$, let $\tilde{z}\left(f^{m}(x)\right)=\tilde{A}^{m}(x) \cdot \hat{z}(x)$. This defines $\tilde{z}$ on $\bigsqcup_{m=1}^{n-1} f^{m}(K)$. We let $\tilde{z}=\hat{z}$ on the rest of $X$. It is easy to see that $\tilde{z}$ is a continuous extension of $z \mid \Lambda$ and that $\tilde{z}$ is an invariant section for $\tilde{A}$. 
To complete the proof, we need to check that $\tilde{A}$ and $A$ are $\|\cdot\|_{\infty}$-close. We have

$$
\begin{aligned}
\mathrm{d}\left(A^{n}(x) \cdot \hat{z}(x), \hat{z}\left(f^{n} x\right)\right) & \leq \mathrm{d}\left(A^{n}(x) \cdot \hat{z}(x), 0\right)+\mathrm{d}\left(0, \hat{z}\left(f^{n} x\right)\right) \\
& \leq \mathrm{d}(\hat{z}(x), 0)+2 \log \left\|A^{n}(x)\right\|+\mathrm{d}\left(0, \hat{z}\left(f^{n} x\right)\right) \\
& \leq 2\left(C_{1}+2 d N \gamma\right)+2 n \gamma \leq[4+2 d(d+2)] n \gamma .
\end{aligned}
$$

Hence, using Lemma 6.2,

$$
\begin{aligned}
\|\tilde{A}-A\|_{\infty} & \leq C\left\|\tilde{A} A^{-1}-\mathrm{Id}\right\|_{\infty} \leq C \sup _{x \in X}\left[\exp \left[\frac{1}{2 n} \mathrm{~d}\left(A^{n}(x) \cdot \hat{z}(x), \hat{z}\left(f^{n} x\right)\right)\right]-1\right] \\
& \leq C\left(e^{[2+d(d+2)] \gamma}-1\right)<\varepsilon .
\end{aligned}
$$

Let us obtain Theorem 5 from the proposition just proved:

Proof of Theorem 5. Assume that $f: X \rightarrow X$ is a minimal homeomorphism with a finite-dimensional non-periodic factor. We claim that if $X$ is finite-dimensional, or there exist at most countably many ergodic invariant measures for $f$, then a generic $A \in C(X, \mathrm{SL}(2, \mathbb{R})) \backslash \mathcal{U} \mathcal{H}$ satisfies

$$
\lim _{n \rightarrow \infty} \sup _{x \in X} \frac{1}{n} \log \left\|A^{n}(x)\right\|=0 .
$$

In the first case, this is the main result of [AB]. In the second case, [Bo] shows that for each ergodic invariant measure $\mu$, for a generic $A \in C(X, \operatorname{SL}(2, \mathbb{R}))$, the Lyapunov exponent relative to $\mu$ vanishes, so taking the (at most countable) intersection, we conclude that for a generic $A \in C(X, \operatorname{SL}(2, \mathbb{R}))$, the Lyapunov exponent vanishes simultaneously for all ergodic invariant measures, and it follows from Proposition 1 in [AB] that (11) holds.

Applying Proposition 6.3 with $\Lambda=\emptyset$, we conclude that any cocycle $A \in$ $C(X, \operatorname{SL}(2, \mathbb{R})) \backslash \mathcal{U} \mathcal{H}$ can be approximated by some $\tilde{A}$ that is conjugate to a cocycle of rotations.

\section{Solving the cohomological equation}

From now on, we assume as usual that $f: X \rightarrow X$ is strictly ergodic with invariant probability measure $\mu$ and has a non-periodic finite-dimensional factor.

Lemma 7.1. Let $T$ be a locally compact separable metric space and let $T^{*} \subset T$ be a closed subset (possibly empty). Let $\varphi \in C^{0}(T \times X, \mathbb{R})$ and $\psi \in C\left(T^{*} \times X, \mathbb{R}\right)$ be such that $\varphi(t, x)=\psi(t, f(x))-\psi(t, x)+c(t)$ for $(t, x) \in T^{*} \times X$, where $c(t)=\int \varphi(t, \cdot) d \mu$. Then, for every continuous function $\varepsilon: T \rightarrow \mathbb{R}_{+}$, there exist $\tilde{\varphi} \in C(T \times X, \mathbb{R})$, coinciding with $\varphi$ on $T^{*} \times X$, and $\tilde{\psi} \in C(T \times X, \mathbb{R})$, coinciding with $\psi$ on $T^{*} \times X$, such that $|\tilde{\varphi}(t, x)-\varphi(t, x)|<\varepsilon(t)$ and $\tilde{\varphi}(t, x)=\tilde{\psi}(t, f(x))-\tilde{\psi}(t, x)+c(t)$ for all $(t, x) \in T \times X$.

The proof of this proposition is somewhat similar to Proposition 6.3, using the abstract Lemma 4.1 and Proposition 5.1. 
Proof. Let us assume first that $T$ is compact. In this case, we may replace the function $\varepsilon$ by a positive number. Let the functions $\varphi$ and $\psi$ be given. Let $S_{j}(t, x)=$ $\sum_{m=0}^{j-1} \varphi\left(t, f^{m}(x)\right)$ and $c(t)=\int \varphi(t, x) d \mu(x)$. Using that $T$ is compact, it is easy to see that $j^{-1} S_{j}(t, x)$ converges to $c(t)$ uniformly over $(t, x) \in T \times X$.

Let $d$ be given by Proposition 5.1. Choose $\delta>0$ with $[2 d(d+2)+1] \delta<\varepsilon$, and choose $j_{0}$ such that $\left|j^{-1} S_{j}(t, x)-c(t)\right|<\delta$ uniformly for $(t, x) \in T \times X$, and $j \geq j_{0}$. Then choose $n \geq j_{0}$ such that $2\|\psi\|_{\infty} / n+[2 d(d+2)+1] \delta<\varepsilon$. (Here $\|\psi\|_{\infty}$ is meant to be 0 in the case that $T^{*}=\emptyset$.) By Proposition 5.1, there is a compact set $K$ that is $d$-mild, $n$-good, and $N$-spanning, where $N=(d+2) n-1$.

Let $\hat{X}=T \times X$, and $\hat{f}: \hat{X} \ni(t, x) \mapsto(t, f(x))$. Consider the skew-product on $\hat{X} \times \mathbb{R}$ over $\hat{f}$ given by

$$
F=F_{\hat{f}, \varphi}^{\mathbb{R}}:(t, x, w) \mapsto(t, f(x), w+\varphi(t, x)-c(t)) .
$$

Let $\Lambda=T^{*} \times X$. By assumption, the map $\psi \in C(\Lambda, \mathbb{R})$ is an invariant section of the skew-product $F \mid \Lambda \times \mathbb{R}$ over $\hat{f} \mid \Lambda$. Let $\psi_{0} \in C(\hat{X}, \mathbb{R})$ be a continuous extension of $\psi$, so that $\left\|\psi_{0}\right\|_{\infty}=\|\psi\|_{\infty}$. (If $T^{*}=\emptyset$, take $\psi_{0} \equiv 0$.) Then $\psi_{0}$ is an almost invariant section for the skew-product $F$ supported outside $\Lambda$. Applying Lemma 4.1 with the pair $(Y, M)=(\mathbb{R},|\cdot|)$, we obtain an almost invariant section $\hat{\psi} \in C(\hat{X}, \mathbb{R})$ supported in int $K$ (i.e., $\varphi(t, x)=\hat{\psi}(t, f(x))-\hat{\psi}(t, x)+c(t)$, provided $x \notin$ int $K$ ) that coincides with $\psi_{0}$ (and hence with $\psi$ ) on $\Lambda$, and

$$
\begin{aligned}
\|\hat{\psi}\|_{\infty} & \leq\left\|\psi_{0}\right\|_{\infty}+d \max _{j \in[n, N]}\left\|F^{j}\right\|_{M}=\|\psi\|_{\infty}+d \max _{j \in[n, N]} \sup _{(t, x)}\left|S_{j}(t, x)-j c(t)\right| \\
& <\|\psi\|_{\infty}+d N \delta .
\end{aligned}
$$

Let us define a function $\tilde{\varphi}: \hat{X} \rightarrow \mathbb{R}$. For each $t \in T, x \in K$, and $0 \leq m<n$, set

$$
\tilde{\varphi}\left(t, f^{m}(x)\right)=\varphi\left(t, f^{m}(x)\right)+\frac{\hat{\psi}\left(t, f^{n}(x)\right)-\hat{\psi}(t, x)-S_{n}(t, x)+n c(t)}{n} .
$$

This defines $\tilde{\varphi}$ on $T \times \bigsqcup_{m=0}^{n-1} f^{m}(K)$; let it equal $\varphi$ in the complement of this set. Then $\tilde{\varphi}$ is continuous and

$$
\|\tilde{\varphi}-\varphi\|_{\infty} \leq 2\|\psi\|_{\infty} / n+[2 d(d+2)+1] \delta<\varepsilon .
$$

Notice that if $t \in T^{*}$, then $\hat{\psi}(t, x)=\psi_{0}(t, x)=\psi(t, x)$ and thus $\tilde{\varphi}(t, x)=\varphi(t, x)$.

Let us define another function $\tilde{\psi}: \hat{X} \rightarrow \mathbb{R}$. For each $t \in T, x \in K$, and $0 \leq m<n$, set

$$
\tilde{\psi}\left(t, f^{m}(x)\right)=\hat{\psi}(t, x)+\sum_{j=0}^{m-1}\left[\tilde{\varphi}\left(t, f^{j}(x)\right)-c(t)\right] .
$$

This defines $\tilde{\psi}$ on $T \times \bigsqcup_{m=0}^{n-1} f^{m}(K)$; let it equal $\hat{\psi}$ in the complement of this set. Then $\tilde{\psi}$ is continuous and $\tilde{\varphi}(t, x)=\tilde{\psi}(t, f(x))-\tilde{\psi}(t, x)+c(t)$ for all $(t, x) \in T \times X$. Also, $\tilde{\psi}(t, x)=\psi(t, x)$ when $t \in T^{*}$. So the functions $\tilde{\varphi}$ and $\tilde{\psi}$ have all the desired properties. 
This concludes the proof in the case where $T$ is compact. Let us now consider the general case. The hypotheses on $T$ imply that there exists an exhaustion of $T$ in the sense that $T=\bigcup_{i=1}^{\infty} T_{i}$ with $T_{i}$ compact and $T_{i} \subset$ int $T_{i+1}$.

We initially define $\tilde{\varphi}$ and $\tilde{\psi}$ on $T_{1} \times X$, by applying the compact case with the data $T_{\text {new }}=T_{1}, T_{\text {new }}^{*}=T_{1} \cap T^{*}, \varphi_{\text {new }}=\varphi\left|T_{1}, \psi_{\text {new }}=\psi\right|\left(T_{1} \cap T^{*}\right), \varepsilon_{\text {new }}=\varepsilon \mid T_{1}$ : this yields functions $\tilde{\varphi}_{\text {new }}, \tilde{\psi}_{\text {new }}: T_{1} \times X \rightarrow \mathbb{R}$, which we take as the definition of $\tilde{\varphi}$ and $\tilde{\psi}$ on $T_{1} \times X$. By construction, all requirements are satisfied when we restrict considerations to $T_{1} \times X$.

Assume we have already defined $\tilde{\varphi}$ and $\tilde{\psi}$ on $T_{i} \times X$ so that all requirements are satisfied when considerations are restricted to $T_{i} \times X$. Choose a continuous function $\varphi_{i}$ : $T_{i+1} \times X \rightarrow \mathbb{R}$ that coincides with $\tilde{\varphi}$ on $T_{i} \times X$ and with $\varphi$ on $\left(T^{*} \cap T_{i+1}\right) \times X$, such that $\int \varphi_{i}(t, x) d \mu(x)=c(t)$ for every $t \in T_{i+1}$ and satisfying $\left|\varphi_{i}(t, x)-\varphi(t, x)\right|<\varepsilon(t)$ for every $(t, x) \in T_{i+1} \times X$. Now apply the compact case again with data $T_{\text {new }}=T_{i+1}$, $T_{\text {new }}^{*}=T_{i} \cup\left(T_{i+1} \cap T^{*}\right), \varphi_{\text {new }}=\varphi_{i}, \psi_{\text {new }}=\tilde{\psi} \mid\left(T_{i} \cup\left(T_{i+1} \cap T^{*}\right)\right)$ and $\varepsilon_{\text {new }}(t)=$ $\varepsilon(t)-\sup _{x \in X}\left|\varphi_{i}(t, x)-\varphi(t, x)\right|$. This yields functions $\tilde{\varphi}_{\text {new }}, \tilde{\psi}_{\text {new }}: T_{i+1} \times X \rightarrow \mathbb{R}$, which we take as the definitions of $\tilde{\varphi}$ and $\tilde{\psi}$ on $T_{i+1} \times X$. Notice that, by construction, the new definitions extend the previous ones, and satisfy all the requirements when we restrict considerations to $T_{i+1} \times X$.

By induction, this procedure yields functions $\tilde{\varphi}, \tilde{\psi}: T \times X \rightarrow \mathbb{R}$. These functions are continuous since they locally coincide with a continuous function obtained in a finite stage of the induction (here we use that $\bigcup$ int $T_{i}=T$ ), and satisfy all the other requirements over $T \times X$ (since those can also be verified at a finite stage of the induction).

Proof of Theorem 8. Let $A \in \mathcal{U H}$, and let $D \in \operatorname{SL}(2, \mathbb{R})$ be such that $L(D)=L(A)$. Set

$$
\Delta(r)=\left(\begin{array}{cc}
e^{r} & 0 \\
0 & e^{-r}
\end{array}\right)
$$

We can assume that $D=\Delta(c)$ for some $c \in \mathbb{R}$. It is not difficult (see, e.g., the proof of Lemma 4 in $[\mathrm{ABD}])$ to show that there are continuous maps $B: X \rightarrow \operatorname{PSL}(2, \mathbb{R})$ and $\varphi: X \rightarrow \mathbb{R}$ such that $A(x)=B(f x)^{-1} \Delta(\varphi(x)) B(x)$ (in $\left.\operatorname{PSL}(2, \mathbb{R})\right)$ and $\int \varphi d \mu=c$.

Apply Lemma 7.1 (with $T=(0,1]$ and $T^{*}=\emptyset$ ) to find continuous functions $\tilde{\varphi}, \tilde{\psi}$ : $(0,1] \times X \rightarrow \mathbb{R}$ such that

$$
\begin{aligned}
& |\tilde{\varphi}(t, x)-\varphi(x)|<\varepsilon(t) \quad \text { where } \varepsilon(t) \rightarrow 0 \text { as } t \rightarrow 0, \\
& \tilde{\varphi}(t, x)=\tilde{\psi}(t, f x)-\tilde{\psi}(t, x)+c .
\end{aligned}
$$

Define

$$
A_{t}(x)=B(f x)^{-1} \Delta(\varphi(t, x)) B(x) \quad \text { and } \quad B_{t}(x)=\Delta(-\tilde{\psi}(t, x)) B(x)
$$

Then $A_{t} \rightarrow A$ as $t \rightarrow 0$ and $A_{t}(x)=B_{t}(f x) D B_{t}(x)^{-1}$, as desired. 


\section{Extending families of cocycles: small Lyapunov exponents}

Let $T$ be a topological space. We say that $A_{t}, t \in T$, is a continuous family of cocycles if $A_{t} \in C(X, \operatorname{SL}(2, \mathbb{R}))$ for every $t \in T$ and the map $T \ni t \mapsto A_{t} \in C(X, \operatorname{SL}(2, \mathbb{R}))$ is continuous. If $\mathcal{B}$ is a subset of $C(X, \operatorname{SL}(2, \mathbb{R}))$ that contains $\left\{A_{t}: t \in T\right\}$ and such that the map $T \ni t \mapsto A_{t} \in \mathcal{B}$ is homotopic to a constant, then we say that that the family of cocycles $A_{t}$ is contractible in $\mathcal{B}$. For example, a continuous family of cocycles $A_{t}$, $t \in \partial[0,1]^{p}$, taking values in a set $\mathcal{B}$ is contractible in $\mathcal{B}$ if and only if there is an extended continuous family of cocycles $A_{t}, t \in[0,1]^{p}$, also taking values in $\mathcal{B}$.

Given a continuous family of cocycles $A_{t}, t \in T$, we say that $z_{t}, t \in T$, is a continuous family of sections if each $t \in T$ corresponds to an invariant section $z_{t} \in C(X, \mathbb{D})$ for the skew-product $F_{f, A_{t}}^{\mathbb{D}}$ (see §6.2), and the map $T \ni t \mapsto z_{t} \in C(X, \mathbb{D})$ is continuous.

The parameter spaces that we use next are the $p$-dimensional cube $[0,1]^{p}$ and its boundary $\partial[0,1]^{p}$. The goal of this section is to prove the following result:

Lemma 8.1. Let $p \geq 1$ and let $\mathcal{B} \subset C(X, \operatorname{SL}(2, \mathbb{R}))$ be a bounded open set. Let $A_{t}$, $t \in \partial[0,1]^{p}$, be a continuous family of cocycles that is contractible in $\mathcal{B}$, and such that $L\left(A_{t}\right)=0$ for all $t \in \partial[0,1]^{p}$. Then, for any $\gamma>0$, we can find a continuous family of cocycles $\hat{A}_{t}, t \in[0,1]^{p}$, taking values in $\mathcal{B}$ and extending the family $A_{t}, t \in \partial[0,1]^{p}$, such that $L\left(\hat{A}_{t}\right)<\gamma$ for every $t \in[0,1]^{p}$.

The proof of Lemma 8.1 requires several preliminaries. A measurable set $Y \subset X$ is called a coboundary if there is a measurable set $Z$ such that $Y=Z \Delta f^{-1}(Z) \mu$-mod 0 . Noncoboundaries are relatively easy to find:

Lemma 8.2 (Corollary 3.5 from $[\mathrm{K}]$ ). If $Z \subset X$ is a positive measure set, then there is a measurable set $Y \subset Z$ that is not a coboundary.

Our interest in non-coboundaries is due to the following fact, already used in [K]:

Lemma 8.3. Let $A: X \rightarrow \operatorname{SL}(2, \mathbb{R})$ be such that $\log \|A\|$ is $\mu$-integrable. Assume that there exist two measurable maps $e^{1}, e^{2}: X \rightarrow \mathbb{P}^{1}$ such that

$$
e^{1}(x) \neq e^{2}(x), \quad A(x) \cdot\left\{e^{1}(x), e^{2}(x)\right\}=\left\{e^{1}(f x), e^{2}(f x)\right\} \quad \text { for } \mu \text {-almost every } x .
$$

If the set $Y=\left\{x \in X: A(x) \cdot e^{1}(x)=e^{2}(f x)\right\}$ is not a coboundary, then the Lyapunov exponent $L(A)$ vanishes.

Proof. Assume that $L(A)>0$, and consider the two Oseledets directions $e^{+}, e^{-}$: $X \rightarrow \mathbb{P}^{1}$. It follows from Oseledets's Theorem that $\left\{e^{1}(x), e^{2}(x)\right\}=\left\{e^{+}(x), e^{-}(x)\right\}$ for almost every $x$. Let $W$ be the set of $x$ where $e^{1}(x)=e^{+}(x)$. Then $W \Delta f^{-1}(W)$ is precisely the set $Y$. Hence it is a coboundary.

If $Z \subset X$ is a measurable set of positive measure, the first-return map $f_{Z}: Z \rightarrow Z$ is defined as $f_{Z}(x)=f^{r_{Z}(x)}(x)$, where $r_{Z}(x)$ is the least positive integer $n$ such that $f^{n}(x) \in Z$. The probability measure $\mu / \mu(Z)$ is invariant and ergodic with respect to $f_{Z}$. If $A: X \rightarrow \operatorname{SL}(2, \mathbb{R})$ is such that $\log \|A\| \in L^{1}(\mu)$, then we define the induced cocycle $A_{Z}: Z \rightarrow \operatorname{SL}(2, \mathbb{R})$ by $A_{Z}(x)=A^{r_{Z}(x)}(x)$. 
Lemma 8.4 (cf. Lemma 2.2 from $[\mathrm{K}]$ ). The Lyapunov exponent of the cocycle $A_{Z}$ over the dynamics $f_{Z}$ and with respect to the ergodic measure $\mu / \mu(Z)$ is equal to $L(A) / \mu(Z)$.

A well-known theorem by Lusin states that a measurable function can be altered in a set of arbitrarily small measure so that it becomes continuous. We will need a parameterized version of this:

Lemma 8.5. Assume $T$ is a compact metric space. Let $\psi: T \times X \rightarrow \mathbb{R}$ be a Borel measurable function such that for every $t \in T$, there is a full measure subset of $X$ consisting of points $x$ with the property that the function $t^{\prime} \mapsto \psi\left(t^{\prime}, x\right)$ is continuous on a neighborhood of $t$. Then for every $\beta>0$, there exists a continuous function $\phi: T \times X \rightarrow \mathbb{R}$ such that for every $t \in T$, the points $x \in X$ where $\phi(t, x) \neq \psi(t, x)$ form a set of measure less than $\beta$.

Proof. For each $t \in T$ and $r>0$, let $\bar{B}(t, r)$ be the closed ball of radius $r$ centered at $t$, and

$$
G(t, r)=\left\{x \in X: t^{\prime} \mapsto \psi\left(t^{\prime}, x\right) \text { is continuous on } \bar{B}(t, r)\right\} .
$$

For every $t \in T$, there is $\rho(t)>0$ such that $\mu(G(t, \rho(t)))>1-\beta / 3$. Take a cover of $T$ by finitely many balls $B_{i}=\bar{B}\left(t_{i}, \rho\left(t_{i}\right)\right)$. Now, for each $i$, let $K_{i} \subset G\left(t_{i}, \rho\left(t_{i}\right)\right)$ be compact with $\mu\left(K_{i}\right)>1-2 \beta / 3$. Consider the mapping $\Psi_{i}: K_{i} \rightarrow C\left(B_{i}, \mathbb{R}\right)$ given by $t^{\prime} \mapsto\left(x \in B_{i} \mapsto \psi\left(t^{\prime}, x\right)\right)$. Take a subset $K_{i}^{\prime} \subset K_{i}$ with $\mu\left(K_{i}^{\prime}\right)>1-\beta$ such that $\Psi_{i} \mid K_{i}^{\prime}$ is continuous. Let $L=\bigcup_{i} B_{i} \times K_{i}^{\prime}$. Then $L$ is compact, $\psi$ restricted to $L$ is continuous, and for each $t$, the set of $x$ such that $(t, x)$ belongs to $L$ has measure at least $1-\beta$. Let $\phi: T \times X \rightarrow \mathbb{R}$ be a continuous extension of $\psi \mid L$, given by Tietze's Extension Theorem. Then $\phi$ has the required properties.

We say that $A_{t}, t \in T$, is an $L^{\infty}$ family of cocycles if $A_{t} \in L^{\infty}(X, \operatorname{SL}(2, \mathbb{R}))$ for every $t \in T$, the map $(t, x) \mapsto A_{t}(x)$ is measurable and $\sup _{t}\left\|A_{t}\right\|_{\infty}$ is finite.

We say that $A_{t}, t \in T$, is an almost-continuous family of cocycles if it is an $L^{\infty}$ family, $T$ is a compact metric space, and for every $t \in T$, there is a full measure set $G_{t} \subset X$ such that for each $x \in G_{t}$, the mapping $t^{\prime} \mapsto A_{t^{\prime}}(x)$ is continuous in a neighborhood of $t$.

We define a weak metric on the set $L^{\infty}(X, \operatorname{SL}(2, \mathbb{R}))$ as follows:

$$
d_{\mathrm{w}}(A, B)=\inf \{\beta>0: \mu\{x \in X:\|A(x)-B(x)\|>\beta\}<\beta\} .
$$

Lemma 8.6. Assume $A_{t}, t \in T$, is an almost-continuous family of cocycles. Let $\lambda=$ $\sup _{t \in T} L\left(A_{t}\right)$, and fix $M>\sup _{t}\left\|A_{t}\right\|_{\infty}$. Then:

(a) $\lim _{n \rightarrow \infty} \sup _{t \in T} n^{-1} \int_{X} \log \left\|A_{t}^{n}\right\| d \mu=\lambda$;

(b) for every $\gamma>\lambda$, there exists $\beta>0$ with the following properties. Assume that $B_{t}$, $t \in T$, is an $L^{\infty}$ family of cocycles such that $\left\|B_{t}\right\|_{\infty}<M$ and $d_{\mathrm{w}}\left(B_{t}, A_{t}\right)<\beta$ for every $t \in T$. Then $L\left(B_{t}\right)<\gamma$ for every $t$.

Proof. The limit in part (a) exists by subadditivity, and is obviously larger than or equal to $\lambda$. Let us prove the reverse inequality. 
Fix $\eta>0$ and take $t \in T$. Let $m \in \mathbb{N}$ be such that $m^{-1} \int \log \left\|A_{t}^{m}\right\| d \mu<\lambda+\eta$. Since $A_{t}$ is an almost-continuous family of cocycles, so is $A_{t}^{m}$. Hence for any $\varepsilon>0$, there exists $\delta>0$ such that

$$
\mu\left\{x \in X:\left\|A_{t^{\prime}}^{m}(x)-A_{t}^{m}(x)\right\|<\varepsilon \text { for all } t^{\prime} \in B(t, \delta)\right\}>1-\varepsilon .
$$

Since the family is essentially bounded, we can find $\delta=\delta(t)>0$ such that $m^{-1} \int \log \left\|A_{t^{\prime}}^{m}\right\| d \mu<\lambda+2 \eta$ for every $t^{\prime} \in B(t, \delta)$. It follows from subadditivity that there exists $\bar{m}=\bar{m}(t) \in \mathbb{N}$ such that

$$
\frac{1}{n} \int \log \left\|A_{t^{\prime}}^{n}\right\| d \mu<\lambda+3 \eta \quad \text { for all } t^{\prime} \in B(t, \delta) \text { and } n \geq \bar{m} .
$$

Now take a cover of $T$ by finitely many balls $B\left(t_{i}, \delta\left(t_{i}\right)\right)$. Let $n_{0}=\max _{i} \bar{m}\left(t_{i}\right)$. Then

$$
\frac{1}{n} \int \log \left\|A_{t}^{n}\right\| d \mu<\lambda+3 \eta \quad \text { for every } t \in T \text { and } n \geq n_{0} .
$$

Since $\eta>0$ is arbitrary, part (a) of the lemma follows.

Let $\varepsilon>0$ be small. There exists $\beta>0$ such that if $B_{t}, t \in T$, is an $L^{\infty}$ family of cocycles with $d_{\mathrm{w}}\left(B_{t}, A_{t}\right)<\beta$ for each $t$, then $d_{\mathrm{w}}\left(B_{t}^{n_{0}}, A_{t}^{n_{0}}\right)<\varepsilon$. Now, if in addition $\left\|B_{t}\right\|_{\infty}<M$ and $\varepsilon$ was chosen small enough, it follows from (12) that

$$
\frac{1}{n_{0}} \int \log \left\|B_{t}^{n_{0}}\right\| d \mu<\lambda+4 \eta
$$

which implies $L\left(B_{t}\right)<\lambda+4 \eta$ for every $t \in T$. This proves part (b).

After these preliminaries, we can prove Lemma 8.1.

Proof of Lemma 8.1. Let us first explain informally the steps of the proof:

(a) We select a bounded cocycle $D$ in $\mathcal{B}$ and a small tower for $f$ along which some products of $D$ are rotations.

(b) By assumption, the given family $A_{t}$ of cocycles can be extended to a family defined in the whole cube, which we also denote by $A_{t}$.

(c) In the crucial step, we find an almost-continuous family of cocycles $B_{t}$ that has zero exponents for all values of the parameter and is uniformly very close to $A_{t}$ everywhere except in the tower. This is done using Lemma 8.3.

(d) We use Lemma 8.5 to approximate $B_{t}$ by a continuous family of cocycles $\tilde{A}_{t}$; by Lemma 8.6 we get $L\left(\tilde{A}_{t}\right)$ small for every $t$.

(e) Since the original family of cocycles $A_{t}, t \in \partial[0,1]^{p}$, has zero exponents, we can interpolate it with the $\tilde{A}_{t}$ family near the boundary of the cube and obtain a continuous family $\hat{A}_{t}, t \in[0,1]^{p}$, that coincides with the initial family in the boundary, and (by semicontinuity) has small exponent in the whole cube.

Now let us give the actual proof.

Since the set $\mathcal{B}$ is bounded and $L\left(A_{t}\right)=0$ for every $t \in \partial[0,1]^{p}$, by part (b) of Lemma 8.6 there is $\beta>0$ such that whenever $A_{t}^{\prime}, t \in \partial[0,1]^{p}$, is an $L^{\infty}$ family of 
cocycles taking values in $\mathcal{B}$ and with $d_{\mathrm{w}}\left(A_{t}^{\prime}, A_{t}\right)<\beta$, we have $L\left(A_{t}^{\prime}\right)<\gamma$ for every $t \in \partial[0,1]^{p}$.

Since the family $A_{t}, t \in \partial[0,1]^{p}$, is contractible in $\mathcal{B}$, we can extend it to a continuous family $A_{t}, t \in[0,1]^{p}$, also taking values in $\mathcal{B}$.

Let $\varepsilon>0$ be very small. (Actual smallness requirements will emerge several times along the proof.)

Let $t_{\mathrm{c}}=(1 / 2, \ldots, 1 / 2)$ be the center of the cube. We may assume that $A_{t_{\mathrm{c}}}=A_{t}$ for some $t \in \partial[0,1]^{p}$. Using Theorem 5 , choose a cocycle $D$ close to $A_{t_{\mathrm{c}}}$ that has an invariant section $z: X \rightarrow \mathbb{D}$. Making if necessary a small perturbation, we can assume that $z$ assumes the same value $z_{0}$ in an open set. By conjugating everything with some fixed matrix (and hence changing $\mathcal{B}, \gamma, \beta$ accordingly), we can assume that $z_{0}=0$. Let $N$ be a large integer. Take a non-empty open set $Z \subset X$ such that its iterates in times $0=n_{0}<n_{1}<\cdots<n_{N}$ are wholly contained in $\{z=0\}$, and moreover $Z \cap f^{i}(Z)=\emptyset$ for $0<i \leq n_{N}+1$. Notice that if $x \in Z$, then $D^{n_{i}}(x) \in \mathrm{SO}(2, \mathbb{R})$ for $0 \leq i \leq N$. By a further perturbation (and reducing $Z$ if necessary), we can assume that in addition to the previous properties, $D \mid f^{i}(Z)$ is constant for $0 \leq i \leq n_{N}$, and equal to the identity matrix for $i=n_{N}$. Reducing $Z$ further, we assume that the tower $\bigcup_{i=0}^{n_{N}-1} f^{i}(Z)$ has measure less than $\beta / 2$. Let $W=f^{n_{N}}(Z)$ and $F=f^{n_{N}}: Z \rightarrow W$. For $x \in W$, let $\ell(x)>0$ be minimal with $f^{\ell(x)}(x) \in Z$, and let $G: W \rightarrow Z$ be given by $G(x)=f^{\ell(x)}(x)$.

Since the set $\mathcal{B}$ is open, we can modify the family $A_{t}, t \in[0,1]^{p}$, near $t=t_{\mathrm{c}}$ so that $A_{t_{\mathrm{c}}}$ equals $D$.

Let $P_{t}: W \rightarrow \mathrm{SL}(2, \mathbb{R})$ be given by $P_{t}(x)=A_{t}^{\ell(x)}(x)$.

Claim. There exists an $L^{\infty}$ family $\tilde{P}_{t}, t \in[0,1]^{p}$ with the following properties:

(a) $\left\|P_{t}^{-1} \tilde{P}_{t}-\operatorname{Id}\right\|_{\infty}<\varepsilon$ for every $t$;

(b) $(t, x) \mapsto \tilde{P}_{t}(x)$ is finite-valued and takes values in $\mathrm{SL}(2, \mathbb{R}) \backslash \mathrm{SO}(2, \mathbb{R})$;

(c) for every $t$, there is a full measure subset of $W$ consisting of points $x$ such that the map $t^{\prime} \mapsto \tilde{P}_{t^{\prime}}(x)$ is constant in a neighborhood of $t$.

Proof. Since $f$ is minimal and $Z$ is open, $\ell(x)$ is bounded and therefore so is the function $P_{t}(x)$. Choose then a large finite set of $\mathrm{SL}(2, \mathbb{R}) \backslash \mathrm{SO}(2, \mathbb{R})$ approximating its image.

Since $(X, \mu)$ is a non-atomic Lebesgue space, we can assume $X$ is the unit interval and $\mu$ is Lebesgue measure. Let $\mathcal{Q}$ be a partition of $\mathbb{R}^{p}$ into small dyadic cubes. Fix a vector $v_{0} \in \mathbb{R}^{p}$ whose coordinates are independent mod $\mathbb{Q}$. For each $x \in[0,1]$, let $\mathcal{Q}(x)$ be the restriction of the partition $x v_{0}+\mathcal{Q}$ to the unit cube. Then every $t \in[0,1]^{p}$ belongs to the interior (relative to $[0,1]^{p}$ ) of an element of the partition $\mathcal{Q}(x)$ for all but finitely many points $x \in X$. Take $\tilde{P}_{t}(x)$ to be constant in each element of $\mathcal{Q}(x)$, and taking values in the large finite set. This proves the claim.

For each $x \in W$, let $e_{t}^{s}(x)$ and $e_{t}^{u}(x)$ be respectively the most contracted and the most expanded directions by $\tilde{P}_{t}(x)$; these two directions are uniquely defined because the matrix is not a rotation, and are orthogonal. Next define $e_{t}^{s}(x)$ and $e_{t}^{u}(x)$ for $x \in Z=G(W)$ by

$$
e_{t}^{*}(x)=\tilde{P}_{t}\left(G^{-1}(x)\right) \cdot e_{t}^{*}\left(G^{-1}(x)\right), \quad * \in\{s, u\} .
$$

These two directions are also orthogonal. 
There are only finitely many possible values for $e_{t}^{u}(x)$ and $e_{t}^{s}(x)$; let $\mathcal{F} \subset \mathbb{P}^{1}$ be the set of all of them. Define a function $\measuredangle: \mathcal{F} \times \mathcal{F} \rightarrow[0, \pi)$ such that $R_{\measuredangle(x, y)} \cdot x=y$.

Apply Lemma 8.2 and choose some set of positive measure $Y \subset Z$ that is not a coboundary. Let

$$
\theta(t, x)= \begin{cases}\measuredangle\left(e_{t}^{u}(x), e_{t}^{s}(F(x))\right) & \text { if } x \in Y, \\ \measuredangle\left(e_{t}^{u}(x), e_{t}^{u}(F(x))\right) & \text { if } x \in Z \backslash Y .\end{cases}
$$

Notice that for every $t$ and for every $x$ in a full measure subset of $X$ (depending on $t$ ), the function $t^{\prime} \mapsto \theta\left(t^{\prime}, x\right)$ is constant in a neighborhood of $t$.

Define

$$
\begin{aligned}
& B_{t}(x) \\
& = \begin{cases}D(x) \cdot R_{\theta\left(t, f^{\left.-n_{i} x\right) / N}\right.} & \text { if } x \in f^{n_{i}}(Z), 0 \leq i<N, \\
D(x) & \text { if } x \in f^{j}(Z), j \in\left\{0,1, \ldots, n_{N}\right\} \backslash\left\{n_{0}, n_{1}, \ldots, n_{N-1}\right\}, \\
A_{t}(x) \cdot\left[P_{t}(x)\right]^{-1} \cdot \tilde{P}_{t}(x) & \text { if } x \in W, \\
A_{t}(x) & \text { otherwise. }\end{cases}
\end{aligned}
$$

Let us list some properties of the family of cocycles $B_{t}, t \in[0,1]^{p}$. First, it is almostcontinuous. Second,

$$
L\left(B_{t}\right)=0 \quad \text { for every } t \in[0,1]^{p} .
$$

Indeed, the induced cocycle $\left(B_{t}\right)_{Z}(x)$ is given by

$$
C_{t}(x)=\tilde{P}_{t}(F(x)) R_{\theta(t, x)}, \quad x \in Z .
$$

(Of course, this cocycle is regarded over the dynamics $G \circ F$, which is the first return map to $Z$.) The pair of measurable directions $e_{t}^{u}, e_{t}^{s}$ is preserved by the action of the cocycle; moreover the directions are swapped precisely for the points in $Y$. Thus Lemma 8.3 gives that for each $t$, the measurable cocycle $\left(G \circ F, C_{t}\right)$ has zero Lyapunov exponent (with respect to the ergodic invariant measure $(1 / \mu(Z)) \mu$ ). Thus (13) follows from Lemma 8.4.

A third property of the family $B_{t}$ is that for each $t, B_{t}(x)$ is close to $A_{t}(x)$ for every $x$ outside a set of small measure. Let us be more precise. Let $\tau(t, x)$ equal $t_{c}$ if $x$ is in the tower $\bigcup_{i=0}^{n_{N}} f^{i}(Z)$ (which has measure less than $\beta / 2$ ), and $t$ otherwise. Then we can write

$$
B_{t}(x)=A_{\tau(t, x)}(x) \cdot E_{t}(x),
$$

where $E_{t}, t \in[0,1]^{p}$, is an almost-continuous family of cocycles such that $\left\|E_{t}(x)-\operatorname{Id}\right\|$ $<\varepsilon$ for all $t$ and $x$. (Here we used that $N$ is large.)

Since $\tau(t, x)$ depends continuously on $t$, we can apply for instance Lemma 8.5 and find a continuous function $\tilde{\tau}:[0,1]^{p} \times X \rightarrow[0,1]^{p}$ such that $\mu\{x \in X: \tilde{\tau}(t, x) \neq \tau(t, x)\}$ is very small for every $t$.

Let $\mathbb{B}_{\varepsilon}$ be the set of $M \in \mathrm{SL}(2, \mathbb{R})$ such that $\|M-\mathrm{Id}\|<\varepsilon$. Since $\varepsilon$ is small, this set is homeomorphic to $\mathbb{R}^{3}$. Thus we can apply Lemma 8.5 and find a continuous map $(t, x) \in[0,1]^{p} \times X \mapsto \tilde{E}_{t}(x) \in \mathbb{B}_{\varepsilon}$ such that $\mu\left\{x \in X: \tilde{E}_{t}(x) \neq E_{t}(x)\right\}$ is uniformly small for every $t$. 
Now define

$$
\tilde{A}_{t}(x)=A_{\tilde{\tau}(t, x)}(x) \cdot \tilde{E}_{t}(x) .
$$

Then $\tilde{A}_{t}$ is a continuous family of cocycles and (because $\varepsilon$ is small) it takes values in $\mathcal{B}$. We can choose $\tilde{\tau}(t, x)$ and $\tilde{E}_{t}(x)$ so that $\sup _{t} d_{\mathrm{W}}\left(\tilde{A}_{t}, B_{t}\right)$ is as small as desired. Therefore, by (13) and of Lemma 8.6(b), we can assume that

$$
L\left(\tilde{A}_{t}\right)<\gamma \quad \text { for every } t \in[0,1]^{p} .
$$

With appropriate choices, we also find that for each $t \in[0,1]^{p}$, the set

$$
G_{t}=\{x \in X: \tilde{\tau}(t, x)=t\} \quad \text { has } \mu\left(G_{t}\right)>1-\frac{3}{4} \beta .
$$

The last step of the proof is to modify the family $\tilde{A}_{t}$ to make it match with $A_{t}$ in the boundary of the cube, while keeping the exponent small. Let $\pi:[0,1]^{p} \backslash\left\{t_{\mathrm{c}}\right\} \rightarrow \partial[0,1]^{p}$ be a retraction. Let $S$ be a neighborhood of $\partial[0,1]^{p}$ inside $[0,1]^{p}$, thin enough so that for every $t \in S, \pi(t)$ is close to $t$ and hence $A_{\pi(t)}$ is close to $A_{t}$. Let $\hat{E}_{t}, t \in[0,1]^{p}$, be a continuous family taking values in $\mathbb{B}_{\varepsilon}$, such that $\hat{E}_{t}(x)=\tilde{E}_{t}(x)$ for $t$ outside $S$, and $\hat{E}_{t}(x)=$ Id for $t \in \partial[0,1]^{p}$. Let $\hat{\tau}:[0,1]^{p} \times X \rightarrow[0,1]^{p}$ be continuous such that $\hat{\tau}(t, x)=\tilde{\tau}(t, x)$ if $t \notin S, \hat{\tau}(t, x)=t$ if $t \in \partial[0,1]^{p}$, and moreover $\hat{\tau}(t, x)=t$ for every $t \in[0,1]^{p}$ and $x \in G_{t}$. Define

$$
\hat{A}_{t}(x)=A_{\hat{\tau}(t, x)}(x) \cdot \hat{E}_{t}(x) .
$$

Then the family $\hat{A}_{t}, t \in[0,1]^{p}$, takes values in $\mathcal{B}$ and continuously extends $A_{t}, t \in$ $\partial[0,1]^{p}$. For $t \in[0,1]^{p} \backslash S$, we have $\hat{A}_{t}=\tilde{A}_{t}$ and therefore $L\left(\hat{A}_{t}\right)<\gamma$. For $t \in S$ and $x \in G_{t}$, we have $\hat{A}_{t}(x)=A_{t}(x) \cdot \hat{E}_{t}(x)$, which is close to $A_{\pi(t)}(x)$. Since $\varepsilon$ is small, we have $d_{\mathrm{w}}\left(\hat{A}_{t}, A_{\pi(t)}\right)<\beta$. By the definition of $\beta$, this implies $L\left(\hat{A}_{t}\right)<\gamma$. This completes the proof of Lemma 8.1.

\section{Extending families of cocycles: boundedness}

In this section, we will strengthen Lemma 8.1, obtaining the following two results:

Proposition 9.1. Let $p \geq 1$, and let $\mathcal{B} \subset C(X, \operatorname{SL}(2, \mathbb{R}))$ be a bounded open set. Let $A_{t}$, $t \in \partial[0,1]^{p}$, be a continuous family of cocycles that is contractible in $\mathcal{B}$ and admits a continuous family of sections $z_{t}, t \in \partial[0,1]^{p}$. Then $z_{t}$ can be extended to a continuous family of sections for an extended family of cocycles $A_{t}, t \in[0,1]^{p}$, that also takes values in $\mathcal{B}$.

Proposition 9.2. Let $p \geq 1$, and let $\mathcal{B} \subset C(X, \operatorname{SL}(2, \mathbb{R}))$ be a bounded open set. Let $A_{t}$, $t \in \partial[0,1]^{p}$, be a continuous family of cocycles that is contractible in $\mathcal{B}$ and such that $A_{t} \notin \mathcal{U H}$ for every $t \in \partial[0,1]^{p}$. Then there exists an extended continuous family of cocycles $A_{t}, t \in[0,1]^{p}$, also taking values in $\mathcal{B}$ and such that the family $A_{t}, t \in(0,1)^{p}$, admits a continuous family of sections. 
Notice that Theorem 4 is an immediate consequence of this proposition with $p=1$. It also follows that the complement of $\mathcal{U H}$ is locally connected. This shows the statement made in Remark 2.2.

We will first prove Proposition 9.1, and then use it together with Theorem 5 to obtain Proposition 9.2. If $A \in C(X, \operatorname{SL}(2, \mathbb{R}))$ and $\varepsilon>0$, we write

$$
\mathcal{B}_{\varepsilon}(A)=\left\{\tilde{A}:\|\tilde{A}-A\|_{\infty}<\varepsilon\right\}
$$

Proof of Proposition 9.1. Let $A_{t}, t \in \partial[0,1]^{p}$, be a continuous family of cocycles admitting a continuous family of sections. Assume that this family is contractible in a bounded open set $\mathcal{B} \subset C(X, \operatorname{SL}(2, \mathbb{R}))$, and thus can be extended to a $\mathcal{B}$-valued family $\bar{A}_{t}, t \in[0,1]^{p}$. Let $\varepsilon>0$ be such that for each $t \in[0,1]^{p}$, the ball with center $\bar{A}_{t}$ and radius $2 \varepsilon$, indicated by $\mathcal{B}_{2 \varepsilon}\left(\bar{A}_{t}\right)$, is contained in $\mathcal{B}$. Let $\gamma>0$ be given by Proposition 6.3, where in place of $f$ we take the homeomorphism of $\hat{X}=X \times[0,1]^{p}$ given by $\hat{f}(x, t)=(f(x), t)$, and take $\Lambda=X \times \partial[0,1]^{p}$, and $C>\sup _{B \in \mathcal{B}}\|B\|_{\infty}$. Next apply Lemma 8.1 to the family $A_{t}, t \in \partial[0,1]^{p}$, and to the reduced set $\mathcal{B}^{\prime}=\bigcup_{t \in[0,1]^{p}} \mathcal{B}_{\varepsilon}\left(\bar{A}_{t}\right)$. We obtain a family $\hat{A}_{t}, t \in[0,1]^{p}$, with values in $\mathcal{B}^{\prime}$ such that $L\left(\hat{A}_{t}\right)<\gamma$ for each $t$. Using compactness of $[0,1]^{p}$ and subadditivity, it is not hard to see that

$$
\lim _{n \rightarrow \infty} \sup _{(x, t) \in X \times[0,1]^{p}} \frac{1}{n} \log \left\|\hat{A}_{t}^{n}(x)\right\|<\gamma .
$$

Thus we can apply Proposition 6.3 and find a continuous family of cocycles $\tilde{A}_{t}, t \in$ $[0,1]^{p}$, that equals $\hat{A}_{t}$ (and hence $A_{t}$ ) when $t \in \partial[0,1]^{p}$, such that $\left\|\tilde{A}_{t}-\hat{A}_{t}\right\|_{\infty}<\varepsilon$ (and thus $\left.\tilde{A}_{t} \in \mathcal{B}\right)$ for each $t$, and that admits a continuous family of sections $\tilde{z}_{t}, t \in[0,1]^{p}$, extending the original $z_{t}, t \in \partial[0,1]^{p}$.

Lemma 9.3. For every $C>0$, there is $\bar{\varepsilon}>0$ such that for every $A \in C(X, \operatorname{SL}(2, \mathbb{R}))$ with $\|A\|_{\infty}<C$ and every $\varepsilon \in(0, \bar{\varepsilon}]$, the following holds: Every continuous family of cocycles $A_{t}, t \in T$, taking values in $\mathcal{B}_{\varepsilon}(A)$ is contractible in $\mathcal{B}_{\varepsilon}(A)$.

Proof. Easy and left to the reader.

Now we are able to prove the second main result of this section:

Proof of Proposition 9.2. Let $A_{t}, t \in \partial[0,1]^{p}$, be a family of cocycles that is contractible in $\mathcal{B}$, such that no $A_{t}$ is uniformly hyperbolic.

Let $V_{n}=\left[2^{-n}, 1-2^{-n}\right]^{p}$. Take $n_{0}$ large (to be determined later). Most of the proof will be devoted to the description of a procedure to extend the family of cocycles $A_{t}$ to the set $[0,1]^{p} \backslash$ int $V_{n_{0}}$, which we call the shell.

For each $n \geq n_{0}$, let $W_{n}=V_{n+1} \backslash$ int $V_{n}$. Cover the set $W_{n}$ by closed cubes $W_{n, i}$ of edge length $2^{-n-1}$ whose interiors are disjoint. Let $\mathcal{P}$ be the family of all those cubes $W_{n, i}$. Consider the sets $K_{0} \subset K_{1} \subset \cdots \subset K_{p}$, where $K_{q}$ is the union of the $q$-dimensional faces of cubes in $\mathcal{P}$. We are going to define $A_{t}$ successively for $t$ in $K_{0}$, $K_{1}, \ldots$, up to $K_{p}$, and so obtain the extension to the shell. 
During this proof, we take in $\mathbb{R}^{p}$ the box distance given by

$$
d\left(\left(t_{i}\right),\left(t_{i}^{\prime}\right)\right)=\max _{i}\left|t_{i}-t_{i}^{\prime}\right| .
$$

Fix a sequence $\varepsilon_{1}>\varepsilon_{2}>\cdots$ converging to 0 such that if $\tau, \tau^{\prime} \in \partial[0,1]^{p}$ are $2^{-n}$-close, then $\left\|A_{\tau}-A_{\tau^{\prime}}\right\|_{\infty}<\varepsilon_{n}$.

Take $t \in K_{0}$ and let us define $A_{t}$. First choose some $\tau=\tau(t) \in \partial[0,1]^{p}$ such that the distance from $t$ to $\tau$ is as small as possible; then that distance equals $2^{-n}$ for some $n \geq 2$. Since $A_{\tau}$ is not uniformly hyperbolic, by Theorem 5 there is a cocycle $B$ with $\left\|B-A_{\tau}\right\|<\varepsilon_{n}$ that admits a continuous section. Set $A_{t}=B$.

The definition of $A_{t}$ proceeds by an inductive procedure. Assume that $A_{t}$ is already defined for $t \in K_{q}$. Fix $F$ to be a $(q+1)$-dimensional face in $K_{q+1}$, and let $B=B(F)$ be its relative boundary, that is, the union of the $q$-dimensional faces in $K_{q}$ that intersect $F$. Choose any point $t_{0}$ in $B$, and let $r=r(B)$ be the diameter of $\left\{A_{t}: t \in B\right\}$ in $C(X, \operatorname{SL}(2, \mathbb{R}))$. If $r$ is sufficiently small, then, by Lemma 9.3 combined with Proposition 9.1, we can extend the continuous family of cocycles $A_{t}, t \in B$ (which takes values in the ball $\left.\mathcal{B}_{2 r}\left(A_{t_{0}}\right)\right)$ and its continuous family of sections to the $(q+1)$-dimensional cube $F$, so that the extended family of cocycles takes values in $\mathcal{B}_{4 r}\left(A_{t_{0}}\right)$. The smallness of $r$ will be proven by induction, of course. In fact, we will prove that there exist small numbers $r_{n}^{(q)}>0$ with $\lim _{n \rightarrow \infty} r_{n}^{(q)}=0$ such that if $F$ as above has diameter $2^{-n}$, then $r(B) \leq r_{n}^{(q)}$.

First consider $q=0$, so $F$ is an edge and $B=\left\{t_{0}, t_{1}\right\}$. If $2^{-n}$ is the edge length, then $t_{0}$ and $t_{1}$ are both within distance $2^{-n+1}$ of the boundary of the unit cube, hence $d\left(t_{i}, \tau\left(t_{i}\right)\right) \leq 2^{-n+1}$ for $i=0,1$. It follows that $d\left(\tau\left(t_{0}\right), \tau\left(t_{1}\right)\right)<2^{-n+3}$ and so

$$
\begin{aligned}
r(B)=\left\|A_{t_{0}}-A_{t_{1}}\right\|_{\infty} & \leq\left\|A_{t_{0}}-A_{\tau\left(t_{0}\right)}\right\|_{\infty}+\left\|A_{\tau\left(t_{0}\right)}-A_{\tau\left(t_{1}\right)}\right\|_{\infty}+\left\|A_{\tau\left(t_{1}\right)}-A_{t_{1}}\right\|_{\infty} \\
& \leq \varepsilon_{n-1}+\varepsilon_{n-3}+\varepsilon_{n-1}<3 \varepsilon_{n-3} .
\end{aligned}
$$

Thus we set $r_{n}^{(0)}=3 \varepsilon_{n-3}$. These numbers will all be small for all $n \geq n_{0}$, provided $n_{0}$ is chosen large enough. This proves the $q=0$ case.

Now let us explain how to find $r_{n}^{(q)}$ assuming $r_{n}^{(q-1)}$ is known. Take a $(q+1)$ dimensional cell $F$; its relative boundary $B=B(F)$ is composed of $2^{q+1}$ cells of dimension $q$. In each of these cells, the variation of $A_{t}$ is at most $4 r_{n}^{(q-1)}$. Hence a crude estimate gives $r(B) \leq 2^{q+3} r_{n}^{(q-1)}=: r_{n}^{(q)}$. Take $n_{0}$ large enough so that these numbers will be small for all $n \geq n_{0}$.

At this point we have defined $A_{t}$ for $t$ in the shell. To check continuity in the boundary of the unit cube, take a sequence $t_{j}$ in $(0,1)^{p} \backslash V_{n_{0}}$ converging to some $\tau \in \partial[0,1]^{p}$. For each $j$, consider a cube of the family $\mathcal{P}$ that contains $t_{j}$, and let $t_{j}^{\prime}$ be one of its vertices. Then $\left\|A_{t_{j}}-A_{t_{j}^{\prime}}\right\|_{\infty} \rightarrow 0$ as $j \rightarrow \infty$ due to the variation estimates. On the other hand, $\tau\left(t_{j}^{\prime}\right) \rightarrow \tau$ and $\left\|A_{\tau\left(t_{j}^{\prime}\right)}-A_{t_{j}^{\prime}}\right\|_{\infty} \rightarrow 0$, therefore $A_{t_{j}} \rightarrow A_{\tau}$.

Now, taking a smaller shell if necessary, we can assume that all the values of $A_{t}$ belong to the open set $\mathcal{B}$. Finally, apply Proposition 9.1 once more to extend continuously the family of cocycles from the shell to the whole unit cube, still taking values in $\mathcal{B}$, and in such a way that in $(0,1)^{p}$, there is a continuous family of sections. This concludes the proof of Proposition 9.2. 


\section{Proof of Theorems 6 and 7}

Let $A \in C_{0}(X, \operatorname{SL}(2, \mathbb{R})) \backslash \mathcal{U H}$ be an unlocked cocycle. Fix a continuous determination of the fibered rotation number $\rho: \mathcal{B}_{\varepsilon_{0}}(A) \rightarrow \mathbb{R}$ on the ball of radius $\varepsilon_{0}$ and center $A$. As proved in Appendix C (see Proposition C.1), we have

$$
\rho\left(R_{-\theta} A\right)<\rho(A)<\rho\left(R_{\theta} A\right) \quad \text { for every sufficiently small } \theta>0 .
$$

The first step is to show the following:

Lemma 10.1. There is a continuous family of cocycles $A_{t}, t \in[-1,1]$, such that $A_{0}=A$,

$$
\rho\left(A_{-t}\right)<\rho(A)<\rho\left(A_{t}\right) \text { for every } t \in(0,1],
$$

and no $A_{t}$ is uniformly hyperbolic.

Proof. Choose $\theta_{n}<0$ to be an increasing sequence close to 0 and converging to 0 , such that $2 \rho\left(R_{\theta_{n}} A\right) \notin G$ for each $n$. The sequence $\rho\left(R_{\theta_{n}} A\right)$ is also strictly increasing (cf. Proposition C.1). So, by continuity of $\rho$, we can find a sequence $\delta_{n}$ decreasing to 0 such that

$\rho\left(R_{\theta_{n-1}} A^{\prime}\right)<\rho\left(R_{\theta_{n}} A^{\prime \prime}\right)<\rho\left(R_{\theta_{n+1}} A^{\prime \prime \prime}\right)<\rho\left(A^{\prime \prime \prime \prime}\right) \quad$ for all $A^{\prime}, A^{\prime \prime}, A^{\prime \prime \prime}, A^{\prime \prime \prime \prime} \in \mathcal{B}_{\delta_{n}}(A)$.

Define an open subset of $C(X, \mathrm{SL}(2, \mathbb{R}))$ by

$$
\mathcal{T}_{n}=\bigcup_{\theta \in\left[\theta_{n}, \theta_{n+1}\right]} R_{\theta} \mathcal{B}_{\delta_{n}}(A) .
$$

Claim. For each $B \in \mathcal{T}_{n}$, there exists a unique $\beta \in\left[-\theta_{n+1}+\theta_{n-1},-\theta_{n}\right]$ such that $\rho\left(R_{\beta} B\right)=\rho\left(R_{\theta_{n+1}} A\right)$. Moreover, the function $B \mapsto \beta$ is continuous.

Proof. Let $B \in \mathcal{T}_{n}$, so $B=R_{\alpha} A^{\prime}$ for some $\alpha \in\left[\theta_{n}, \theta_{n+1}\right]$ and $A^{\prime} \in \mathcal{B}_{\delta_{n}}(A)$. Then

$$
\rho\left(R_{-\theta_{n+1}+\theta_{n-1}} B\right) \leq \rho\left(R_{\theta_{n-1}} A^{\prime}\right)<\rho\left(R_{\theta_{n+1}} A\right)<\rho\left(A^{\prime}\right) \leq \rho\left(R_{-\theta_{n}} B\right) .
$$

By the Intermediate Value Theorem, there is $\beta \in\left[-\theta_{n+1}+\theta_{n-1},-\theta_{n}\right]$ such that $\rho\left(R_{\beta} B\right)=\rho\left(R_{\theta_{n+1}} A\right)$. Uniqueness is a consequence of Proposition C.1 and the fact that $2 \rho\left(R_{\theta_{n+1}} A\right) \notin G$. Continuity follows.

For each $n$, consider the family of cocycles $R_{\theta} A, \theta \in\left\{\theta_{n+1}, \theta_{n}\right\}$. It is obviously contractible in $\mathcal{T}_{n}$. Thus we can apply Proposition 9.2 to find a continuous one-parameter family of cocycles with values in $\mathcal{T}_{n} \cap \mathcal{U} \mathcal{H}^{c}$, joining $R_{\theta_{n}} A$ to $R_{\theta_{n+1}} A$. By concatenation, we find a continuous family of cocycles $A_{t}, t \in\left[\theta_{1}, 0\right]$, such that

$$
A_{\theta_{n}}=R_{\theta_{n}} A, \quad t \in\left[\theta_{n}, \theta_{n+1}\right] \Rightarrow A_{t} \in \mathcal{T}_{n} \cap \mathcal{U} \mathcal{H}^{c} .
$$

Let

$$
t_{n}=\inf \left\{t \in\left[\theta_{n}, \theta_{n+1}\right]: \rho\left(A_{n, t}\right)=\rho\left(R_{\theta_{n+1}} A\right)\right\} .
$$

Now let us define a "correction" function $\beta:\left[\theta_{1}, 0\right] \rightarrow \mathbb{R}$. Define $\beta(t)=0$ for $t \in\left[\theta_{n}, t_{n}\right]$. For each $t \in\left[t_{n}, \theta_{n+1}\right]$, let $\beta(t)$ be given by the claim above such that $\rho\left(R_{\beta(t)} A_{n, t}\right)=\rho\left(R_{\theta_{n+1}} A\right)$. Finally, put $\beta(0)=0$. Then $\beta$ is well-defined and continuous. 
Now consider the continuous family of cocycles $\tilde{A}_{t}=R_{\beta(t)} A_{t}$. We have $\tilde{A}_{t} \in \mathcal{U} \mathcal{H}^{c}$ for each $t$ (indeed, for $t \in\left[t_{n}, \theta_{n+1}\right]$, this follows from $\left.2 \rho\left(\tilde{A}_{t}\right) \notin G\right)$. Also, in each interval $\left[\theta_{n}, \theta_{n+1}\right]$ we have $\rho\left(\tilde{A}_{t}\right) \leq \rho\left(R_{\theta_{n+1}} A\right)$, so $\rho\left(\tilde{A}_{t}\right)<\rho(A)$ for every $t<0$.

Applying an entirely analogous procedure, we find a family $\tilde{A}_{t}$ for small non-negative $t$ such that $A_{0}=A$ and $\rho\left(\tilde{A}_{t}\right)>\rho(A)$ for $t>0$. By reparameterizing, we find the desired family.

Next we prove the following strengthening of Lemma 10.1:

Lemma 10.2. There is a continuous family of cocycles $A_{t}, t \in[-1,1]$, such that $A_{0}=A$,

$$
\rho\left(A_{-t}\right)<\rho(A)<\rho\left(A_{t}\right) \text { for every } t \in(0,1],
$$

and the family $A_{t}, t \in[-1,1] \backslash\{0\}$, admits a continuous family of sections.

Proof. Let $A_{t}, t \in[-1,1]$, be the continuous family of cocycles given by the previous lemma. We can assume it takes values in a small neighborhood of $A$. Consider the continuous family $B_{t}, t \in \partial[0,1]^{2}$, defined by $B_{\left(t_{1}, t_{2}\right)}=A_{t_{1}}$. Since these cocycles are not uniformly hyperbolic, we can apply Proposition 9.2 to find an extended family $B_{t}$, $t \in[0,1]^{2}$, such that the restricted family $B_{t}, t \in(0,1)^{2}$, is continuously reducible to rotations. Since $\rho\left(B_{\left(t_{1}, 0\right)}\right)>\rho(A)$ for each $t_{1} \in(0,1]$, we can find a continuous function $h:(0,1] \rightarrow(0,1 / 2]$ such that $\rho\left(B_{\left(t_{1} / 2, h\left(t_{1}\right)\right)}\right)>\rho(A)$ for each $t_{1}$. That is, $\tilde{A}_{t}=B_{(t / 2, h(t))}, t \in(0,1]$, defines a continuous family of cocycles that admits a continuous family of sections, satisfies $\rho\left(\tilde{A}_{t}\right)>\rho(A)$ for each $t$, and $\lim _{t \rightarrow 0} \tilde{A}_{t}=A$. In the same way we find the desired cocycles for negative parameters.

In the next step we find a family depending on two parameters:

Lemma 10.3. There exists a family $A_{t}, t \in[0,1]^{2}$, such that $A_{(0,0)}=A$,

$$
\rho\left(A_{(0, s)}\right)<\rho(A)<\rho\left(A_{(s, 0)}\right) \quad \text { for } s \in(0,1],
$$

and the restricted family $A_{t}, t \in[0,1]^{2} \backslash\{(0,0)\}$, admits a continuous family of sections.

Proof. Let $A_{t}^{\prime}, t \in[-1,1]$, be given by Lemma 10.2, so $\rho\left(A_{-t}^{\prime}\right)<\rho(A)<\rho\left(A_{t}^{\prime}\right)$ for $t>0$, and $A_{t}^{\prime}, t \in[-1,1] \backslash\{0\}$, admits a continuous family of sections $z_{t}^{\prime}$.

By Proposition 9.1, we can find a family $A_{t}^{\prime \prime}, t \in[-1,1]$, admitting a continuous family of sections $z_{t}^{\prime \prime}$, such that $A_{ \pm 1}^{\prime \prime}=A_{ \pm 1}^{\prime}$ and $z_{ \pm 1}^{\prime \prime}=z_{ \pm 1}^{\prime}$. Next define $A_{t}$ for $t \in$ $\partial[0,1]^{2}$ by

$$
A_{\left(t_{1}, t_{2}\right)}= \begin{cases}A_{t_{1}-t_{2}}^{\prime} & \text { if } t_{1}=0 \text { or } t_{2}=0 \\ A_{t_{1}-t_{2}}^{\prime \prime} & \text { if } t_{1}=1 \text { or } t_{2}=1 .\end{cases}
$$

Define analogously the family of sections $z_{t}, t \in \partial[0,1]^{2} \backslash\{(0,0)\}$.

Consider the set $\Gamma=\bigcup_{n=1}^{\infty} \partial[0,1 / n]^{2}$. Using Proposition 9.1 (with $p=1$ ) infinitely many times, find an extended continuous family of cocycles $A_{t}, t \in \Gamma$, such that the cocycles $A_{t}, t \in \Gamma \backslash\{(0,0)\}$, admit a continuous family of sections that extends the previously defined $z_{t}$. 
Now consider the regions $[0,1 / n]^{2} \backslash(0,1 /(n+1))^{2}$; each is homeomorphic to a square, and $A_{t}, B_{t}$ are defined in the boundary. Applying Proposition 9.1 (with $p=2$ ) we extend $A_{t}, z_{t}$ to each of those holes. This can be done so that $\lim _{t \rightarrow(0,0)} A_{t}=A$. This gives the desired family of cocycles.

Now we need a purely topological result:

Lemma 10.4. Let $\theta:[0,1]^{2} \rightarrow \mathbb{R}$ be a continuous function. Assume that $\theta(0,0)=0$ and $\theta(0, s)<0<\theta(s, 0)$ for every $0<s \leq 1$. Let $K=\theta^{-1}(0)$ and let $U \subset(0,1]^{2}$ be a neighborhood of $K \backslash\{(0,0)\}$. Then there exists a path $\gamma:(0,1] \rightarrow U$ such that $\lim _{s \rightarrow 0} \gamma(s)=(0,0)$.

Proof. Let $\varepsilon:(0,1] \rightarrow(0,1]$ be a continuous increasing function such that for every $t \in K \backslash\{(0,0)\}, B_{\varepsilon(|t|)}(t) \cap[0,1]^{2} \subset U$.

For every $t \in K \backslash\{(0,0)\}$, choose a closed dyadic square $\tilde{D}_{t}$ containing $t$ of diameter at least $\varepsilon(|t|) / 10$ and at most $\varepsilon(|t|) / 5$, and let $D_{t}$ be the union of all closed dyadic squares of the same diameter that intersect $\tilde{D}_{t}$. Let $W=\bigcup_{t \in K \backslash\{0\}} D_{t}$.

Let $I=\left\{t=\left(t_{1}, t_{2}\right) \in[0,1]^{2}: t_{1}+t_{2}=1 / 2\right\}$. Then $I$ intersects $W$ on finitely many of its connected components, which we denote by $S_{i}$. We claim that one of them accumulates at $(0,0)$. Indeed, if this is not the case, let $T$ be the union of the boundaries of the $S_{i}$ intersected with $\Delta=\left\{t=\left(t_{1}, t_{2}\right) \in[0,1]^{2}: t_{1}+t_{2} \leq 1 / 2\right\}$. Then there exists a path joining $(0,1 / 2)$ to $(1 / 2,0)$ that is contained everywhere in $\left(I \backslash \bigcup S_{i}\right) \cup T$. But the sign of $\theta$ is constant along this path, a contradiction.

Let $S$ be such a connected component that accumulates at $(0,0)$. Write $S$ as a countable union of dyadic squares, so that any of them does not contain another. Order such squares with the property that $D_{i} \cap D_{i+1} \neq \emptyset$. Refine this sequence so that no squares are repeated. Join the centers of the squares by straight segments. This gives the desired path.

Now we can finish the proof of Theorems 6 and 7:

Proof of Theorems 6 and 7. Consider the continuous family of cocycles $\tilde{A}_{t}, t \in[0,1]^{2}$, given by Lemma 10.3; for $t \neq(0,0)$, one can write

$$
\tilde{A}_{t}(x)=\tilde{B}_{t}(f x) R_{2 \pi \theta(t, x)} \tilde{B}_{t}(x)^{-1},
$$

where $\tilde{B}_{t} \in C(X, \operatorname{SL}(2, \mathbb{R})), t \in[0,1]^{2} \backslash\{(0,0)\}$, is a continuous family of conjugacies and $\theta:[0,1]^{2} \times X \rightarrow \mathbb{R}$ is continuous, with $\int \theta(0, s, x) d \mu(x)<$ $\int \theta(0,0, x) d \mu(x)<\theta(s, 0, x)$ for $0<s \leq 1$. Here, we write $\int \theta(0,0, x) d \mu(x)$ for $\lim _{t \rightarrow(0,0)} \int \theta(t, x) d \mu(x)$. By the definition of the fibered rotation number, $\int \theta(0,0, x) d \mu(x)=\rho(A) \bmod G^{\prime}\left(\right.$ where $G^{\prime}$ is a subgroup of $G$, see $\left.\S 1.4\right)$.

Let $g=\alpha-\int \theta(0,0, x) d \mu(x)$ in the case of Theorem 6 , and $g=2 \alpha-$ $2 \int \theta(0,0, x) d \mu(x)$ in the case of Theorem 7. In either case, $g \in G$, so we may write $g=\int \phi d \mu$ for some continuous function $\phi: X \rightarrow \mathbb{R}$ such that $\phi=\psi \circ f-\psi$ for some continuous $\psi: X \rightarrow \mathbb{R} / \mathbb{Z}$. In the case of Theorem 6 , we may thus replace $\tilde{B}_{t}(x)$ by $\tilde{B}_{t}(x) R_{2 \pi \psi(x)}$ and $\theta(t, x)$ by $\theta(t, x)+\phi(t, x)$, that is, we may actually assume that $g=0$. 
In the case of Theorem 7, we may proceed similarly, replacing $\tilde{B}_{t}(x)$ by the PSL $(2, \mathbb{R})$ valued $\tilde{B}_{t}(x) R_{\psi(x)}$, and $\theta(t, x)$ by $\theta(t, x)+\phi(t, x) / 2$ : we may thus also assume that $g=0$ in this case. From now on, we assume that $\alpha=\int \theta(0,0, x) d \mu(x)$.

Apply Lemma 7.1 with $T=[0,1]^{2} \backslash\{(0,0)\}, \varphi=\theta \mid(T \times X), T^{*}=\emptyset$ and $\varepsilon$ so small that $\varepsilon(0, s)<\int \alpha-\theta(0, s, x) d \mu(x), \varepsilon(s, 0)<\int \theta(s, 0, x)-\alpha d \mu(x)$ and $\left\|\tilde{B}_{t}\right\|_{\infty}^{2} \varepsilon(t) \rightarrow 0$ as $t \rightarrow(0,0)$. Let $\tilde{\varphi}$ and $\tilde{\psi}$ be as in the lemma. We now define $\hat{A}_{t}(x)=$ $\tilde{B}_{t}(f(x)) R_{2 \pi \tilde{\varphi}(t, x)} \tilde{B}_{t}(x)^{-1}$ for $t \in[0,1]^{2} \backslash\{(0,0)\}$ and $\hat{A}_{0}=A$; this is a continuous family at $t=(0,0)$ since $\left\|\hat{A}_{t}-\tilde{A}_{t}\right\|_{\infty}<2 \pi \varepsilon(t)\left\|\tilde{B}_{t}\right\|_{\infty}^{2}$ for $t \in[0,1]^{2} \backslash\{(0,0)\}$. Since $\tilde{\varphi}(t, x)$ is cohomologous to $c(t)=\int \theta(t, x) d \mu(x)=\rho\left(\tilde{A}_{t}\right)$, we can write $\hat{A}_{t}(x)=$ $\hat{B}_{t}(f(x)) R_{2 \pi c(t)} \hat{B}_{t}(x)^{-1}$ (where the $\psi$ 's are absorbed by $\hat{B}$ ).

Now choose some function $\varepsilon_{1}(t)>0$ that goes to zero as $t \rightarrow(0,0)$. By Lemma 10.4, there exists a continuous path $\gamma:(0,1] \rightarrow(0,1]^{2}$ whose range is contained in the set of $t$ 's for which $\left\|\hat{A}_{t}(x)-\hat{B}_{t}(f(x)) R_{2 \pi \alpha} \hat{B}_{t}(x)^{-1}\right\|<\varepsilon_{1}(t)$. (The latter set is open and contains $K=c^{-1}(\alpha)$.) Thus, we may set $A_{s}(x)=\hat{B}_{\gamma(s)}(f(x)) R_{2 \pi \alpha} \hat{B}_{\gamma(s)}(x)^{-1}$. By construction, this family has the desired properties.

Remark 10.5. It follows from the proof that if $\alpha=\rho_{f, A} \bmod G^{\prime}$, then the maps $B_{t}$ can be taken in $C_{0}(X, \operatorname{SL}(2, \mathbb{R}))$ (i.e., homotopic to a constant). Conversely, if the conclusion of Theorem 6 holds with $B_{t} \in C_{0}(X, \operatorname{SL}(2, \mathbb{R}))$, then it is straightforward to show that $\rho_{f, A}=\alpha \bmod G^{\prime}$.

Remark 10.6. A cocycle can be accessed by a continuous path of unlocked cocycles with the same rotation number if and only if it is unlocked itself. Indeed, the "only if" part is an immediate consequence of the definitions. On the other hand, notice that if two cocycles in $C_{0}(X, \operatorname{SL}(2, \mathbb{R}))$ are conjugate via a map also in $C_{0}(X, \operatorname{SL}(2, \mathbb{R}))$ then ${ }^{9}$ they have the same type (locked / unlocked / semi-locked). Thus, given an unlocked cocycle $A$, applying Theorem 6 (and Remark 10.5) with $\alpha=\rho_{f, A}$ we obtain a continuous path of unlocked cocycles with the same rotation number.

\section{Appendix A. Connectedness considerations}

Recall that $C_{0}(X, \operatorname{SL}(2, \mathbb{R}))$ indicates the subset of $C(X, \operatorname{SL}(2, \mathbb{R}))$ formed by maps that are homotopic to a constant. The rotation number can be viewed as a map $C_{0}(X, \operatorname{SL}(2, \mathbb{R})) \rightarrow \mathbb{R} / G^{\prime}$

Proposition A.1. If two cocycles have the same rotation number ( $\left.\bmod G^{\prime}\right)$, then it is possible to connect them by a continuous path with constant rotation number ( $\left.\bmod G^{\prime}\right)$.

Proof. Let $A^{\prime}, A^{\prime \prime} \in C_{0}(X, \operatorname{SL}(2, \mathbb{R}))$ be two cocycles such that $\rho_{f, A^{\prime}}=\rho_{f, A^{\prime \prime}} \bmod G^{\prime}$. Fix once and for all a lift $F^{\prime}: X \times \mathbb{R} \rightarrow X \times \mathbb{R}$ such that $e^{2 \pi i F_{2}^{\prime}(x, t)}$ is a positive real multiple of $A^{\prime}(x) \cdot e^{2 \pi i t}$. This determines the fibered rotation number of $\left(f, A^{\prime}\right)$ as some $a \in \mathbb{R}$. Moreover, for any contractible space $Y$ and any continuous map $y \in Y \mapsto A_{y} \in$

\footnotetext{
9 In fact, it is not necessary that the conjugacy is homotopic to a constant; see Proposition C.4.
} 
$C_{0}(X, \operatorname{SL}(2, \mathbb{R}))$ such that $A_{y_{0}}=A^{\prime}$ for some $y_{0} \in Y$, there exists a unique continuous function $\rho: Y \rightarrow \mathbb{R}$ such that $\rho(y)=\rho_{f, A_{y}} \bmod G^{\prime}$ and $\rho\left(y_{0}\right)=a$. We also call $\rho$ a continuous determination of the rotation number. (In fact, the previous use of this terminology is a particular case.)

Take any continuous path $t \in[0,1] \mapsto A_{t} \in C_{0}(X, \operatorname{SL}(2, \mathbb{R}))$ with $A_{0}=A^{\prime}$ and $A_{1}=A^{\prime \prime}$. Consider the continuous determination of the rotation number $\rho:[0,1] \rightarrow$ $\mathbb{R}$ with $\rho(0)=a$. Then $\rho(1)-\rho(0) \in G^{\prime}$, thus there exists $\phi \in C(X, \mathbb{Z})$ such that $\rho(1)-\rho(0)=\int \phi d \mu$. Extend the path of cocycles to the interval $[0,2]$ by defining $A_{t}(x)=R_{-2 \pi(t-1) \phi(x)} A^{\prime \prime}(x)$ for $t \in[1,2]$. Considering the corresponding extension $\rho:[0,2] \rightarrow \mathbb{R}$, we have $\rho(2)=\rho(0)=a$.

Now consider the two-parameter family $(t, \theta) \in[0,2] \times \mathbb{R} \mapsto R_{\theta} A_{t} \in$ $C_{0}(X, \operatorname{SL}(2, \mathbb{R}))$ and the associated continuous determination of the rotation number $\rho:[0,2] \times \mathbb{R} \rightarrow \mathbb{R}$ such that $\rho(0,0)=a$, and thus $\rho(0,2)=a$. Notice that $\rho(t, \theta)$ is non-decreasing as a function of $\theta$, and $\rho(t, \theta+2 \pi)=\rho(t, \theta)+1$.

We claim that the function $f(t)=\inf \{\theta \in \mathbb{R}: \rho(t, \theta)=a\}$ is continuous. Indeed, fix any sequence $t_{n}$ in [0,2] converging to some $t$ and such that $\beta=\lim f\left(t_{n}\right)$ exists. Let $\alpha=f(t)$. Then $\rho(t, \beta)=\lim \rho\left(t_{n}, \beta\right) \leq a=\rho(t, \alpha)$. It follows that $\beta \leq \alpha$. Moreover, if this inequality is strict, then $\rho(t, \theta)$ is constant equal to $a$ for $\theta$ in the interval $(\beta, \alpha)$ and thus by the first part of Proposition C.1, $R_{(\alpha+\beta) / 2} A_{t}$ is uniformly hyperbolic. In particular $\rho(s,(\alpha+\beta) / 2)=a$ for every $s$ sufficiently close to $t$. This implies that $\rho(t, \beta) \leq \lim \rho\left(t_{n}, \beta\right) \leq \lim \rho\left(t_{n},(\alpha+\beta) / 2, t_{n}\right)=a$, and thus $\beta \geq f(t)$, a contradiction.

It now follows that the rotation number is constant $\bmod G^{\prime}$ along the continuous path of cocycles $t \in[0,2] \mapsto R_{\min (0, f(t))} A_{t}$, concluding the proof.

Proposition A.2. If $A_{0}, A_{1} \in C_{0}(X, \operatorname{SL}(2, \mathbb{R})) \backslash \mathcal{U H}$, then we can join these cocycles by a continuous path $t \in[0,1] \mapsto A_{t} \in C(X, \mathrm{SL}(2, \mathbb{R})) \backslash \mathcal{U H}$. Moreover, if $A_{0}$ and $A_{1}$ have the same rotation number $\left(\bmod G^{\prime}\right)$, then we can take the path with constant rotation number $\left(\bmod G^{\prime}\right)$.

Proof. The first assertion is a trivial consequence of Proposition 9.2 with $p=1$.

To prove the second assertion, it suffices to show that for any cocycle $A \in$ $C_{0}(X, \operatorname{SL}(2, \mathbb{R})) \backslash \mathcal{U H}$ and any $\alpha=\rho_{f, A} \bmod G$, there is a path joining $A$ and $R_{2 \pi \alpha}$ along which the rotation number is constant.

Given such $A$ and $\alpha$, apply Theorem 6 and find continuous paths $A_{t}, t \in[0,1]$, and $B_{t}, t \in(0,1]$, in $C(X, \mathrm{SL}(2, \mathbb{R}))$ such that $A_{0}=A$ and $A_{t}(x)=B_{t}(f(x)) R_{2 \pi \alpha} B_{t}(x)^{-1}$.

Since $\operatorname{SO}(2, \mathbb{R})$ is a deformation retract of $\operatorname{SL}(2, \mathbb{R})$, there exists a homotopy $h_{t}$ : $\operatorname{SL}(2, \mathbb{R}) \rightarrow \operatorname{SL}(2, \mathbb{R}), t \in[0,1]$, between the identity $h_{0}$ and an $\mathrm{SO}(2, \mathbb{R})$-valued $\operatorname{map} h_{1}$.

The rotation number is constant along the path

$$
A_{t+1}(x)=h_{t}\left(B_{1}(f x)\right) R_{2 \pi \alpha}\left[h_{t}\left(B_{1}(x)\right)\right]^{-1}, \quad t \in[0,1],
$$

joining $A_{1}$ and the $\mathrm{SO}(2, \mathbb{R})$-valued cocycle $A_{2}$. The latter is homotopic to a constant as a map $X \rightarrow \operatorname{SL}(2, \mathbb{R})$ and thus (using the deformation retraction) also as a map $X \rightarrow$ $\mathrm{SO}(2, \mathbb{R})$. It follows that we can write $A_{2}(x)=R_{\theta_{0}(x)}$ where $\theta_{0}$ is real-valued. Let $\theta_{t}$, 
$t \in[0,1]$, be a homotopy in $C(X, \mathbb{R})$ between $\theta_{0}$ and the constant $2 \pi \alpha$ such that $\int \theta_{t} d \mu$ does not depend on $t$. Define $A_{2+t}(x)=R_{\theta_{t}(x)}$ for $t \in[0,1]$. Then $A_{t}, t \in[0,3]$, is a path joining $A$ and $R_{2 \pi \alpha}$ along which the rotation number is constant.

On the other hand, the set of uniformly hyperbolic cocycles with a given rotation number is not necessarily connected:

Proposition A.3. There exists a strictly ergodic homeomorphism $f$ of a finite-dimensional compact space $X$ and there exist two cocycles $A_{0}, A_{1} \in C_{0}(X, \operatorname{SL}(2, \mathbb{R}))$ with the same rotation number that lie in distinct path-connected components of $\mathcal{U H}$.

Remark A.4. One could raise the question of whether it is possible to add an extra parameter in Theorem 3 and obtain the following statement: "If $A_{s}, s \in[0,1]$, is a path in $C_{0}(X, \operatorname{SL}(2, \mathbb{R}))$ with $2 \rho_{f, A_{s}}$ constant and in $G$, then there is a continuous family of cocycles $A_{s, t},(s, t) \in[0,1]^{2}$, such that $A_{s, 0}=A_{s}$ and $A_{s, t} \in \mathcal{U H}$ for $t>0$." However, it follows from Propositions A.1 and A.3 that such a statement is false in general. (On the other hand, it is possible to add an extra parameter in Theorem 4: we have done that already in Proposition 9.2.)

To prove Proposition A.3 we will need the following well-known lemma. Lacking a suitable reference, we give a proof of it.

Lemma A.5. Let $\alpha \in \mathbb{R} \backslash \mathbb{Q}$ and $\beta \in \mathbb{R}$. For a generic continuous $\phi: \mathbb{R} / \mathbb{Z} \rightarrow \mathbb{R}$ with $\int \phi(x) d x=\beta$, the map ${ }^{10} f_{\alpha, \phi}: \mathbb{R}^{2} / \mathbb{Z}^{2} \rightarrow \mathbb{R}^{2} / \mathbb{Z}^{2}$ given by $f_{\alpha, \phi}(x, y)=(x+\alpha$, $y+\phi(x))$ is strictly ergodic. ${ }^{11}$

Proof. Let $h_{i}: \mathbb{R}^{2} / \mathbb{Z}^{2} \rightarrow \mathbb{R}, i \geq 1$, be a dense sequence in $C\left(\mathbb{R}^{2} / \mathbb{Z}^{2}, \mathbb{R}\right)$. Let $U_{n}$ be the set of all continuous $\phi$ with average $\beta$ such that for every ergodic invariant measure $\mu$ for $f_{\alpha, \phi}$, we have $\mid \int h_{n} d \mu-\int h_{n} d$ Leb $\mid<1 / n$.

Clearly, if $\phi \in \bigcap U_{n}$, then $\phi$ is uniquely ergodic. So we just have to show that all $U_{n}$ are dense. Let $\phi_{0}$ be any trigonometric polynomial. We wish to find $\phi$ close to $\phi_{0}$ that belongs to $U_{n}$. Now, clearly $\phi_{0}(x)=\psi_{0}(x+\alpha)-\psi_{0}(x)+\beta$ for some trigonometric polynomial.

For an arbitrary continuous function $g: \mathbb{R} / \mathbb{Z} \rightarrow \mathbb{R}$, let $\mu_{g}$ be the probability measure on $\mathbb{R}^{2} / \mathbb{Z}^{2}$ that projects to Lebesgue measure in the first factor and is supported on the graph of $x \mapsto x+g(x)$. Consider the sequence of functions $\delta_{k}: \mathbb{R} / \mathbb{Z} \rightarrow \mathbb{R}$ such that $\delta_{k}(x)$ is twice the distance between $k x$ and $\mathbb{Z}$. It is readily seen that $\mu_{\delta_{k}}$ converges in weak-* sense to Leb. It follows that for every continuous function $g, \mu_{g+\delta_{k}}$ converges to Leb (which equals the push-forward of Leb under $(x, y) \mapsto(x, y+g(x)))$.

In particular, $\mu_{\psi_{0}+\delta_{k}} \rightarrow$ Leb. Let $p / q$ be a continued fraction approximant of $\alpha$ and take $\psi=\psi_{0}+\delta_{q}$. Let $\phi(x)=\psi(x+\alpha)-\psi(x)$. Then any invariant measure for $f_{\alpha, \phi}$ is a vertical translate of $\mu_{\psi}$, that is, it is of the form $\left(T_{t}\right)_{*} \mu_{\psi}$ where $T_{t}(x, t)=(x, y+t)$. It follows that all of them are close to Leb, so if we take $q$ sufficiently large, we will have

\footnotetext{
10 Maps of such form are called Anzai skew-products after [An].

11 It is well-known that ergodic Anzai skew-products are in fact strictly ergodic (see [Fu]), but this will not be used below.
} 
$\phi \in U_{n}$. Finally, one computes $\left\|\phi-\phi_{0}\right\|_{\infty} \leq 2|q \alpha-p|<1 / q$, so taking $q$ large also ensures that $\phi$ is close to $\phi_{0}$.

Proof of Proposition A.3. By the previous lemma, there exists a strictly ergodic homeomorphism $f=f_{\alpha, \phi}$ of the torus $\mathbb{R}^{2} / \mathbb{Z}^{2}$ with $\int \phi\left(x_{1}\right) d x_{1}=\alpha \in \mathbb{R} \backslash \mathbb{Q}$. Let $\psi$ : $\mathbb{R} / \mathbb{Z} \rightarrow \mathbb{R}$ be continuous and such that $\psi\left(x_{1}+\alpha\right)-\psi\left(x_{1}\right)$ is uniformly close to $\phi\left(x_{1}\right)-\alpha$. (This can be done easily using the remark after Lemma 3 in [ABD].) Let $u: \mathbb{R}^{2} / \mathbb{Z}^{2} \rightarrow \mathbb{R} / \mathbb{Z}$ be given by

$$
u\left(x_{1}, x_{2}\right)=x_{1}-x_{2}+\psi\left(x_{1}\right) .
$$

Thus $u\left(f\left(x_{1}, x_{2}\right)\right)-u\left(x_{1}, x_{2}\right)=\psi\left(x_{1}+\alpha\right)-\psi\left(x_{1}\right)+\alpha-\phi\left(x_{1}\right)$, which is close to 0 . Notice that $u$ is not homotopic to a constant. Define two elements of $C\left(\mathbb{R}^{2} / \mathbb{Z}^{2}, \operatorname{SL}(2, \mathbb{R})\right)$ by

$$
A_{0}\left(x_{1}, x_{2}\right)=\left(\begin{array}{cc}
2 & 0 \\
0 & 1 / 2
\end{array}\right), \quad A_{1}\left(x_{1}, x_{2}\right)=R_{u\left(f\left(x_{1}, x_{2}\right)\right)}\left(\begin{array}{cc}
2 & 0 \\
0 & 1 / 2
\end{array}\right) R_{-u\left(x_{1}, x_{2}\right)}
$$

These cocycles are uniformly hyperbolic and have the same rotation number (because $u \circ f-u$ lifts to a function of zero mean). But no path $A_{t}, t \in[0,1]$, joining $A_{0}$ and $A_{1}$ can be wholly contained in $\mathcal{U H}$, because the respective expanding directions $e^{u}\left(A_{0}\right), e^{u}\left(A_{1}\right)$ : $\mathbb{R}^{2} / \mathbb{Z}^{2} \rightarrow \mathbb{P}^{1}$ are not homotopic.

\section{Appendix B. Minimal examples with many persistently closed gaps}

In [Be2, Question 4], the question of genericity of Cantor spectrum is asked in a more general context than strictly ergodic dynamical systems. The Gap Labeling Theorem holds, in fact, for any homeomorphism of a compact metric space, and Bellissard asked whether the presence of periodic orbits could be the only obstruction to the genericity of Cantor spectrum (when it is allowed at all by the Gap Labeling Theorem). Though we have answered this question affirmatively in our more restricted setting, the answer to the original question is in fact negative, and as we will see even a slight departure from unique ergodicity (keeping minimality) may already prevent Cantor spectrum.

We will consider the well-known Furstenberg examples (see [Fu, p. 585] or [Pa, §5.5]) of minimal, but non-uniquely ergodic skew-products of the form $f(x, y)=(x+\alpha, y+$ $\phi(x)), \phi \in C\left(X=\mathbb{R}^{2} / \mathbb{Z}^{2}, \mathbb{R}\right), \alpha \in \mathbb{R} \backslash \mathbb{Q}$. For these examples, $G=\mathbb{Z} \oplus \alpha \mathbb{Z}$ is independent of the choice of the invariant measure.

Since $\mathcal{L}$ is dense in $(0,1)$, the Gap Labeling Theorem does not prevent Cantor spectrum for a Furstenberg example, and in fact there are choices of the sampling function exhibiting Cantor spectrum. ${ }^{12}$ However, the next theorem shows that Cantor spectrum (and hence the converse of the Gap Labeling Theorem) is not generic for such dynamics.

\footnotetext{
12 Indeed, the converse of the Gap Labeling Theorem holds for generic potentials which depend only on the first coordinate: this case reduces to the strictly ergodic dynamics $x \mapsto x+\alpha$ which is covered by Corollary 2 .
} 
Theorem 9. Let $f$ be a Furstenberg example.

(a) For an open and dense set of $v \in C(X, \mathbb{R}), \Sigma_{f, v}$ is not a Cantor set.

(b) For a generic set of $v \in C(X, \mathbb{R})$, int $\Sigma_{f, v}$ is dense in $\Sigma_{f, v} .{ }^{13}$

(c) The interior of $C_{0}(X, \operatorname{SL}(2, \mathbb{R})) \backslash \mathcal{U H}$ is dense in $C_{0}(X, \operatorname{SL}(2, \mathbb{R})) \backslash \mathcal{U H}$.

Proof. We start with the third statement. Notice that if $A \in C_{0}(X, \operatorname{SL}(2, \mathbb{R})) \cap \overline{\mathcal{U H}}$, the fibered rotation number (viewed as an element of $\mathbb{R} / G^{\prime}=\mathbb{R} / \mathbb{Z}$ ) is independent of the invariant measure. ${ }^{14}$

If $A \in C_{0}(X, \operatorname{SL}(2, \mathbb{R})) \cap C(X, \operatorname{SO}(2, \mathbb{R}))$, it has the form $A(x)=R_{\phi(x)}$ with $\phi: X \rightarrow \mathbb{R}$ continuous, and the fibered rotation number is independent of the invariant measure if and only if $\int_{X} \phi d \mu$ is. Thus for an open and dense set of $A \in$ $C_{0}(X, \mathrm{SL}(2, \mathbb{R})) \cap C(X, \mathrm{SO}(2, \mathbb{R})), A \notin \overline{\mathcal{U H}}$. By Theorem 5 , if $A$ is not uniformly hyperbolic, it can be approximated by a cocycle which is bounded, and hence by one in the complement of $\mathcal{U H}$. This proves (c).

Using Lemma 1.3, (c) implies that for $v_{0} \in C(X, \mathbb{R})$ and $E \in \Sigma_{f, v_{0}}$, since $A_{E, v_{0}} \notin$ $\mathcal{U H}$, we can perturb $v_{0}$ to $v$ such that $A_{E, v} \notin \overline{\mathcal{U H}}$. Then $E \in \operatorname{int} \Sigma_{f, \tilde{v}}$ for every $\tilde{v}$ in a neighborhood of $v$. This gives (a). A simple Baire category argument allows us to conclude (b).

\section{Appendix C. Uniform hyperbolicity and the rotation number}

In this section we discuss in more detail the relationship between the behavior of the rotation number in a neighborhood of a cocycle that is homotopic to a constant and properties of the cocycle. Recall the definitions of a locked/semi-locked/unlocked cocycle, which were given in Section 2.

Proposition C.1. Let $A \in C(X, \operatorname{SL}(2, \mathbb{R}))$ be homotopic to a constant, and fix a continuous determination of the fibered rotation number $\rho: \mathcal{V} \rightarrow \mathbb{R}$ on a neighborhood of $A$. Then:

(a) The function $r_{A}: \theta \mapsto \rho\left(R_{\theta} A\right)$, defined on an open interval around 0 , is locally constant at $\theta=0$ if and only if $A \in \mathcal{U H}$. Consequently, the cocycle $A$ is:

- locked if $R_{A}$ is locally constant at $\theta=0$;

- semi-locked if $\theta=0$ is in the boundary of a plateau of $r_{A}$;

- unlocked if the value $\rho(A)$ does not correspond to a plateau of $r_{A}$.

(b) The cocycle A is uniformly hyperbolic if and only if it is locked.

(c) If the cocycle $A$ is semi-locked, it can be accessed by uniformly hyperbolic cocycles of the form $A_{\theta}=R_{\theta} A$, where $\theta$ runs through a (non-degenerate) interval of the form $[0, \varepsilon]$ or $[-\varepsilon, 0]$.

(d) If A takes values in $\mathrm{SO}(2, \mathbb{R})$, or if $2 \rho(A) \notin G$, then $A$ is unlocked.

Before giving the proof of this proposition, let us first establish the following lemma.

\footnotetext{
13 It is reasonable to expect that for generic $v, \partial \Sigma_{f, v}$ is a Cantor set (thus $\Sigma_{f, v}$ is not generically a finite union of intervals).

14 It is enough to check this for $A \in C_{0}(X, \operatorname{SL}(2, \mathbb{R})) \cap \mathcal{U H}$ : consider $\rho(A)$ as a continuous function of the convex set of invariant measures, which (since $A \in \mathcal{U H}$ ) takes values in $\frac{1}{2} \mathbb{Z}+\frac{\alpha}{2} \mathbb{Z}$.
} 
Lemma C.2. For any $\varepsilon>0$, there exist $c>0$ and $\lambda>1$ with the following properties. Let $v$ be a non-zero vector in $\mathbb{R}^{2}$. Assume $A_{0}, A_{1}, \ldots, A_{n-1}$ are matrices in $\operatorname{SL}(2, \mathbb{R})$ such that

$$
\left\|A_{n-1} \cdots A_{1} A_{0}(v)\right\|<c \lambda^{n}\|v\| .
$$

Then for any $w \in \mathbb{R}^{2} \backslash\{0\}$, there exists $\theta \in[-\varepsilon, \varepsilon]$ such that the vectors

$$
R_{\theta} A_{n-1} R_{\theta} A_{n-2} \cdots R_{\theta} A_{0} R_{\theta}(v) \text { and } w
$$

are collinear.

A set $I \subset \mathbb{P}^{1}$ is called an interval if it is non-empty, connected, and its complement contains more than one point. If $I \subset \mathbb{P}^{1}$ is an open interval, there is a Riemannian metric $\|\cdot\|_{I}$ on $I$, called the Hilbert metric, which is uniquely characterized by the following properties:

- any invertible linear map $A: \mathbb{R}^{2} \rightarrow \mathbb{R}^{2}$ induces an isometry between $I$ and $A(I)$ with the corresponding Hilbert metrics;

- if $I \subset \mathbb{P}^{1}$ is the projectivization of $\left\{(1, y) \in \mathbb{R}^{2}: y \in(0, \infty)\right\}$, then the Hilbert metric is given by $\frac{d y}{y}$.

If $J \Subset I$ are open intervals, then the metric $\|\cdot\|_{I}$ restricted to $J$ is smaller than $\|\cdot\|_{J}$ times a factor $\tanh \left(\operatorname{diam}_{I}(J) / 4\right)<1$ (Garrett Birkhoff's formula).

Proof of Lemma C.2. We can assume that $\varepsilon<\pi / 2$, otherwise the conclusion is trivial. Let $|\cdot|$ indicate the Euclidean (angle) metric on $\mathbb{P}^{1}$ (so that the length of $\mathbb{P}^{1}$ is $\pi$ ). Fix constants $0<C_{1}<C_{2}$ (depending only on $\varepsilon$ ) such that for any open interval $I$ whose Euclidean length $|I|$ is between $\varepsilon$ and $\pi-\varepsilon$, we have:

$$
\begin{aligned}
& \|\mathbf{u}\|_{I} \geq C_{1}|\mathbf{u}| \quad \text { for every } \mathbf{u} \text { tangent to } I ; \\
& \|\mathbf{u}\|_{I} \leq C_{2}|\mathbf{u}| \quad \text { if } \mathbf{u} \text { is a tangent vector at the midpoint of } I .
\end{aligned}
$$

Also fix a constant $\tau>1$ such that if $J \Subset I$ are open intervals with the same midpoint and $|J|+\varepsilon=|I| \leq \pi-\varepsilon$, then

$$
\|\mathbf{u}\|_{J} \geq \tau\|\mathbf{u}\|_{I} \quad \text { for every } \mathbf{u} \text { tangent to } J .
$$

Now take $A_{0}, \ldots, A_{n-1} \in \operatorname{SL}(2, \mathbb{R})$ and a unit vector $v \in \mathbb{R}^{2}$. Let $\bar{v} \in \mathbb{P}^{1}$ correspond to $v$. Assume that

$$
\left\{R_{\theta} A_{n-1} \cdots A_{1} R_{\theta} A_{0} R_{\theta}(\bar{v}): \theta \in[-\varepsilon, \varepsilon]\right\} \neq \mathbb{P}^{1} ;
$$

we want to show that $\left\|A_{n-1} \cdots A_{0}\right\|$ is exponentially large.

Let us define some open intervals $I_{0}, I_{1}, \ldots, I_{n} \subset \mathbb{P}^{1}$. Let $I_{0}$ be the open interval with midpoint $\bar{v}$ and length $\left|I_{0}\right|=\varepsilon$. Define inductively $I_{k}$ as the open interval with the same midpoint as $A_{k-1}\left(I_{k-1}\right)$ and length $\left|I_{k}\right|=\left|A_{k-1}\left(I_{k-1}\right)\right|+\varepsilon$. It follows from (14) and the fact that the matrices $A_{j}$ preserve orientation that $I_{k}$ is indeed an interval, with endpoints $R_{-\varepsilon / 2} A_{k-1} \cdots R_{-\varepsilon / 2} A_{0} R_{-\varepsilon / 2}(\bar{v})$ and $R_{\varepsilon / 2} A_{k-1} \cdots R_{\varepsilon / 2} A_{0} R_{\varepsilon / 2}(\bar{v})$, and length $\left|I_{k}\right| \leq \pi-\varepsilon$. Therefore for any vector $\mathbf{u}$ tangent to $I_{k-1}$, we have $\left\|D A_{k-1}(\mathbf{u})\right\|_{I_{k}}$ $\leq \tau^{-1}\|\mathbf{u}\|_{I_{k-1}}$. 
Let $\mathbf{w}$ be a non-zero tangent vector at $\bar{v}$, and write $B=A_{n-1} \cdots A_{0}$. Then

$$
|D B(\mathbf{w})| \leq C_{1}^{-1}\|D B(\mathbf{w})\|_{I_{n}} \leq C_{1}^{-1} \tau^{-n}\|\mathbf{w}\|_{I_{0}} \leq C_{1}^{-1} C_{2} \tau^{-n}|\mathbf{w}| .
$$

Since $\|B(v)\|^{-2}=|D B(\mathbf{w})| /|\mathbf{w}|,{ }^{15}$ we see that $\|B(v)\|$ is exponentially large, as desired.

Proof of Proposition C.1. We begin by proving the first statement in part (a). Obviously, $\rho$ is locally constant around $A$ if it is uniformly hyperbolic. Let us prove the other direction. As usual, fix a lift $F_{2}: X \times \mathbb{R} \rightarrow \mathbb{R}$ of $A$. The nearby lift of $R_{\theta} A$ is $F_{2}+\theta$, hence the rotation number is given by

$$
\rho\left(R_{\theta} A\right)=\lim _{n \rightarrow \infty} \frac{1}{n} \int\left[\left(F_{2}+\theta\right)^{n}(x, t)-t\right] d \mu(x) \quad(t \text { arbitrary }),
$$

where $\left(F_{2}+\theta\right)^{n}(x, t)$ is the second coordinate of the $n$-th iterate of $(x, t) \mapsto$ $\left(f(x), F_{2}(x, t)+\theta\right)$.

Assume that $(f, A)$ is not uniformly hyperbolic. Fix $\varepsilon>0$. We will show that $\rho\left(R_{\varepsilon} A\right)>\rho\left(R_{-\varepsilon} A\right)$. Let $c>0$ and $\lambda>1$ be given by Lemma C.2, depending on $\varepsilon$. There exist $n_{0} \in \mathbb{N}$ and $x \in X$ such that $\left\|A^{n_{0}}(x)\right\|<c \lambda^{n_{0}}$. Let $G$ indicate the (positive measure) set of such points $x$. Then Lemma C.2 implies that

$$
\left(F_{2}+\varepsilon\right)^{n_{0}}(x, t)-\left(F_{2}-\varepsilon\right)^{n_{0}}(x, t)>1 / 2 \quad \text { for all } x \in G, t \in \mathbb{R} .
$$

When $x \notin G$, the left-hand side is positive. Since orbits under $f^{n_{0}}$ visit $G$ with an average frequency $\mu(G)$, we conclude that

$$
\begin{aligned}
\rho\left(R_{\varepsilon} A\right)-\rho\left(R_{-\varepsilon} A\right) & =\lim _{k \rightarrow \infty} \frac{1}{n_{0} k} \int\left[\left(F_{2}+\varepsilon\right)^{n_{0} k}(x, t)-\left(F_{2}-\varepsilon\right)^{n_{0} k}(x, t)\right] d \mu(x) \\
& \geq \frac{\mu(G)}{2 n_{0}},
\end{aligned}
$$

as we wanted to show. This proves the first part of (a).

Let us now prove the remaining parts of (a). Suppose $A$ is such that $r_{A}: \theta \mapsto \rho\left(R_{\theta} A\right)$ is constant on $[0, \varepsilon]$. By what we just proved, it follows that $R_{\varepsilon / 2} A \in \mathcal{U H}$. Take a neighborhood $V$ of $A$ such that $R_{\varepsilon / 2} B \in \mathcal{U H}$ and $\rho\left(R_{\varepsilon / 2} B\right)=\rho\left(R_{\varepsilon / 2} A\right)=\rho(A)$ for every $B \in V$. Since $r_{A}$ is non-decreasing, we get $\rho(B) \leq \rho\left(R_{\varepsilon / 2} B\right)=\rho(A)$ and hence $\rho$ attains a local maximum at $A$. Analogously, if $A$ is such that $\theta \mapsto \rho\left(R_{\theta} A\right)$ is constant on $[-\varepsilon, 0]$, then $\rho$ attains a local minimum at $A$. This establishes the remaining three implications in part (a).

Parts (b), (c), and (d) follow readily from (a). (To see the second part of (d), note that if $A$ is not unlocked, then $\theta \mapsto \rho\left(R_{\theta} A\right)$ is constant equal to $\rho_{0}$ on an interval $I$ of the form $[0, \varepsilon]$ or $[-\varepsilon, 0]$. Thus, $R_{\theta} A$ is uniformly hyperbolic for all $\theta \in \operatorname{int} I$ and hence $2 \rho(A) \in G$.)

\footnotetext{
15 Indeed, the slice of the unit disk corresponding to a small angle $|\mathbf{w}|$ around $v$ is a region of $\mathbb{R}^{2}$ with area $\|v\|^{2}|\mathbf{w}|$ that is mapped by $B$ to a region of area $\|B(v)\|^{2}|D B(\mathbf{w})|+\mathcal{O}\left(|\mathbf{w}|^{2}\right)$; but $B$ is area-preserving.
} 
Remark C.3. As is apparent from the proof, Proposition C. 1 holds in the more general setting where $f$ is a homeomorphism of a compact space with a fully supported invariant probability measure $\mu$ and the rotation numbers are taken with respect to this measure (see $[H, \S 5.8 .2])$.

Proposition C.4. Conjugate cocycles have the same type (locked/semi-locked/unlocked).

Proof. Let $B \in C(X, \operatorname{SL}(2, \mathbb{R}))$ be such that $x \mapsto B(f(x)) B(x)^{-1}$ is homotopic to a constant and let $\Phi: C_{0}(X, \operatorname{SL}(2, \mathbb{R})) \rightarrow C_{0}(X, \operatorname{SL}(2, \mathbb{R}))$ be given by $\Phi(A)(x)=$ $B(f(x)) A(x) B(x)^{-1}$.

Let $\mathcal{U}$ be an open connected subset of $C_{0}(X, \operatorname{SL}(2, \mathbb{R}))$ such that there exists a continuous choice of lifts $A \in \mathcal{U} \mapsto F_{A, 2} \in C(X \times \mathbb{R}, \mathbb{R})$. These lifts induce determinations $\rho: \mathcal{U} \rightarrow \mathbb{R}$ and $\rho^{\prime}: \Phi(\mathcal{U}) \rightarrow \mathbb{R}$ of the fibered rotation number. We are going to show that there exists $c \in \mathbb{R}$ such that $\rho^{\prime}(\Phi(A))=\rho(\Phi(A))+c$ for every $A \in \mathcal{U}$. The proposition follows immediately.

Let also $\Theta: X \times \mathbb{R} \rightarrow \mathbb{R}$ be a measurable lift of $B$ that is continuous in the second variable, but not necessarily in the first variable, such that $\Theta(X \times\{0\})$ is bounded. It follows that there exists a bounded measurable function $\psi: \mathcal{U} \times X \rightarrow \mathbb{Z}$ such that

$$
F_{\Phi(\tilde{A}), 2}(f(x), \Theta(x, t))=\Theta\left(f(x), F_{\tilde{A}, 2}(x, t)\right)+\psi(\tilde{A}, x) .
$$

Thus $\rho^{\prime}(\Phi(\tilde{A}))-\rho(A)=\int \psi(\tilde{A}, x) d \mu(x)$. On the other hand, $\psi(\tilde{A}, x)$ is easily seen to be continuous in the first variable, and since $\mathcal{U}$ is connected, it depends only on the second variable.

\section{Appendix D. Proof of the projection lemma}

Let us now prove Lemma 1.3. In the case where tr $A$ does not vanish identically, this result is contained in Lemma 9 of [ABD]. We will explain the necessary changes to deal with the remaining case.

We wish to show that an arbitrary continuous $\operatorname{SL}(2, \mathbb{R})$ perturbation $\tilde{A}$ of the constant cocycle $A$ is conjugate to a Schrödinger perturbation (with a conjugacy depending continuously on the perturbation, and equal to id at $A$ ). On the other hand, given any fixed compact set of non-empty interior $K \subset X$, Lemma 10 of [ABD] states that under the assumption of minimality, $\tilde{A}$ is conjugate to a perturbation $\hat{A}$ such that $\hat{A}=A$ outside $K$.

So it is enough to describe how to conjugate such a localized perturbation. Since $X$ has at least four points, we can choose $K$ admitting a compact neighborhood $K^{\prime}$ such that $f^{i}\left(K^{\prime}\right) \cap K^{\prime}=\emptyset$ for $1 \leq i \leq 3$. Notice that $A^{4}=\mathrm{id}$, so $\hat{A}^{4}$ is close to id. Define $E=\sup _{x \in X}\left\|\hat{A}^{4}(x)-\mathrm{id}\right\|^{1 / 2}$.

For each $x \in K^{\prime}$, there are unique $E_{i}(x) \in \mathbb{R}, 1 \leq i \leq 4$, such that $E_{3}(x)=$ $E \phi(x)$ and $S_{E_{4}(x)} \cdots S_{E_{1}(x)}=\hat{A}^{4}(x)$, where for $t \in \mathbb{R}, S_{t}$ denotes the Schrödinger matrix $\left(\begin{array}{cc}t & -1 \\ 1 & 0\end{array}\right)$. Indeed, write $\hat{A}^{4}(x)=R_{-\pi / 2} \hat{A}(x)=\mathrm{id}+\left(\begin{array}{ll}a(x) & b(x) \\ c(x) & d(x)\end{array}\right)$, and notice that $E_{1}(x), E_{2}(x)$ and $E_{4}(x)$ must be given by

$$
E_{1}(x)=\frac{b(x)-E \phi(x)}{1+d(x)}, \quad E_{2}(x)=-\frac{d(x)}{E \phi(x)}, \quad E_{4}(x)=\frac{c(x)+E_{2}(x)}{1-d(x)},
$$


with the expression for $E_{2}(x)$ being evaluated as 0 whenever $E \phi(x)=0$. These expressions immediately imply that the maps $E_{i}: K^{\prime} \rightarrow \mathbb{R}$ are continuous and vanish in $\partial K^{\prime}$.

We now define $\Phi(\hat{A})$ as follows. Outside $\bigcup_{i=0}^{3} f^{i}\left(K^{\prime}\right)$, we set $\Phi(\hat{A})=\hat{A}=A$. For $x \in K^{\prime}$, we define $\Phi(\hat{A})\left(f^{i}(x)\right)=S_{E_{i+1}(x)}, 0 \leq i \leq 3$. It is easy to see that $\Phi$ depends continuously on $\hat{A}$ in a neighborhood of $A$.

By construction, $\Phi(\hat{A})^{4}(x)=\hat{A}^{4}(x)$ for $x \in K$. Define $\Psi(\hat{A})=$ id outside $\bigcup_{i=0}^{3} f^{i}\left(K^{\prime}\right), \Psi(\hat{A})\left(f^{i}(x)\right)=\Phi(\hat{A})^{i}(x) \hat{A}^{i}(x)^{-1}$ for $x \in K^{\prime}$ and $1 \leq i \leq 3$. Then $\Psi$ is a continuous function of $\hat{A}$ with $\Psi(\hat{A})(f(x)) \cdot \hat{A}(x) \cdot(\Psi(\hat{A})(x))^{-1}=\Phi(\hat{A})(x)$ for every $x \in X$. This completes the proof of Lemma 1.3.

Remark D.1. Like [ABD, Lemma 9], Lemma 1.3 is also valid in smooth settings. We only need to be careful in two steps. First, we should choose $\phi$ to be a smooth function. Second, we should replace the definition of $E=E(\hat{A})$, which should be "much larger than $d$ " for our argument to work, by something that takes into account the derivatives of $d$. In the case of $C^{r}$ cocycles with finite $r$, we could take $E=\|d\|_{C^{r}}^{1 / 2}$, and it is straightforward to check that both $\Phi$ and $\Psi$ are $C^{r}$-valued continuous maps defined in a $C^{r}$-neighborhood of $A$. In the case $r=\infty$, we can take

$$
E=-\frac{1}{\log \sup _{x \in K^{\prime}}\left\|\hat{A}^{4}(x)-\mathrm{id}\right\|}
$$

whenever $\hat{A} \neq A$, and let $E=0$ when $\hat{A}=A$. With this definition, $\Phi$ and $\Psi$ are $C^{0}$ valued continuous maps in a $C^{0}$-neighborhood of $A$, but their restrictions to $C^{s+1}$ are $C^{s}$-valued continuous maps for every finite $s$ (using the convexity inequality $\|\cdot\|_{C^{s}} \leq$ $K_{s}\left(\|\cdot\|_{\left.C^{0}\|\cdot\|_{C^{s+1}}^{s}\right)^{1 /(s+1)}}\right.$; see $\left.[\mathrm{N}]\right)$, so their restrictions to $C^{\infty}$ are $C^{\infty}$-valued continuous maps.

\section{Appendix E. Mixing dynamics and Cantor spectrum}

In [Fa], Fayad proved that there exists a real-analytic time change $f^{t}$ of an irrational flow on $\mathbb{R}^{3} / \mathbb{Z}^{3}$ which is mixing. Thus $f^{t}$ is generated by a vector field of the form $u \alpha$, where the coordinates of $\alpha \in \mathbb{R}^{3}$ are rationally independent and $u: \mathbb{R}^{3} / \mathbb{Z}^{3} \rightarrow \mathbb{R}$ is a positive real-analytic function. By general properties of time changes (see [Pa, §5.1]), to any measure $\mu_{0}$ which is invariant by the flow generated by $\alpha$, we can associate an $f^{t}$ invariant measure which is absolutely continuous with respect to $\mu_{0}$ and has density $1 / u$, and all $f^{t}$-invariant measures are obtained in this way. Since irrational flows are uniquely ergodic, the flow $f^{t}$ is uniquely ergodic as well. We will denote by $\mu$ the unique $f^{t}$ invariant probability measure.

Proposition E.1. Let $f=f^{1}$ be the time one map of the flow $f^{t}$. Then $f$ is mixing, strictly ergodic, and the range $G$ of the Schwartzman asymptotic cycle of $f$ is dense in $\mathbb{R}$.

Proof. Obviously $f$ is mixing. If $\mu^{\prime}$ is any invariant probability measure for $f$, then $\int_{0}^{1} f_{*}^{t} \mu^{\prime} d t$ is an $f^{t}$-invariant probability measure, so it coincides with $\mu$. On the other 
hand, for every $\varepsilon>0, \mu_{\varepsilon}^{\prime}=\varepsilon^{-1} \int_{0}^{\varepsilon} f_{*}^{t} \mu^{\prime} d t$ is an $f$-invariant probability measure absolutely continuous with respect to $\mu$. Obviously $\mu_{\varepsilon}^{\prime} \rightarrow \mu^{\prime}$ as $\varepsilon \rightarrow 0$, and since $f$ is mixing, $f_{*}^{n} \mu_{\varepsilon}^{\prime} \rightarrow \mu$ as $n \rightarrow \infty$ (in both cases the limits are taken in the weak-* topology). Since $\mu_{\varepsilon}^{\prime}$ is $f$-invariant, we conclude that $\mu_{\varepsilon}^{\prime}=\mu$ for every $\varepsilon>0$, and hence $\mu^{\prime}=\mu$. Thus $f$ is uniquely ergodic. Since supp $\mu=\mathbb{R}^{3} / \mathbb{Z}^{3}$, unique ergodicity implies strict ergodicity.

Given $k \in \mathbb{Z}^{3}$, let $\psi: \mathbb{R}^{3} / \mathbb{Z}^{3} \rightarrow \mathbb{R} / \mathbb{Z}$ be given by $\psi(x)=\langle x, k\rangle$, and let $\phi(x)=$ $\int_{0}^{1} u\left(f^{t}(x)\right) d t\langle\alpha, k\rangle$. Then $\phi(x)=\psi(f(x))-\psi(x) \bmod 1$ and $g=\int_{\mathbb{R}^{3} / \mathbb{Z}^{3}} \phi d \mu \in G$. Recalling that $\mu$ is an $f^{t}$-invariant probability measure with density proportional to $1 / u(x)$, we get

$$
\begin{aligned}
\int_{\mathbb{R}^{3} / \mathbb{Z}^{3}} \phi(x) d \mu(x) & =\int_{0}^{1} \int_{\mathbb{R}^{3} / \mathbb{Z}^{3}} u\left(f^{t}(x)\right) d \mu(x) d t\langle\alpha, k\rangle=\int_{\mathbb{R}^{3} / \mathbb{Z}^{3}} u(x) d \mu(x)\langle\alpha, k\rangle \\
& =C^{-1}\langle\alpha, k\rangle,
\end{aligned}
$$

where $C=\int_{\mathbb{R}^{3} / \mathbb{Z}^{3}} u(x)^{-1} d x>0$ is the normalization constant making $\mu$ a probability measure. Since the coordinates of $\alpha$ are rationally independent, this shows that $G$ contains a free Abelian subgroup of rank 3 , so $G$ is dense in $\mathbb{R}$.

It follows from the proposition and Corollary 2 that Schrödinger operators arising from mixing strictly ergodic dynamics can have Cantor spectrum, thus answering negatively [Be1, Problem 5].

Acknowledgments. We are indebted to Jean Bellissard, Russell Johnson, and Barry Simon for useful comments and hints to the literature. J. B. would like to thank Rice University for its hospitality. D. D. would like to express his gratitude to IMPA and PUC-Rio for their hospitality and financial support through PRONEX-Dynamical Systems and Faperj. This research was partially conducted during the period A. A. served as a Clay Research Fellow. J. B. was partially supported by a grant from CNPq-Brazil. D. D. was supported in part by NSF grants DMS-0653720 and DMS-0800100. We benefited from CNPq and Procad/CAPES support for traveling.

\section{References}

[An] Anzai, H.: Ergodic skew product transformations on the torus. Osaka Math. J. 3, 83-99 (1951) Zbl 0043.11203 MR 0040594

[At] Athanassopoulos, K.: Some aspects of the theory of asymptotic cycles. Exposition. Math. 13, 321-336 (1995) Zbl 0837.54027 MR 1358211

[AB] Avila, A., Bochi, J.: A uniform dichotomy for generic $\operatorname{SL}(2, \mathbb{R})$ cocycles over a minimal base. Bull. Soc. Math. France 135, 407-417 (2007) Zbl pre05366168 MR 2430187

[ABD] Avila, A., Bochi, J., Damanik, D.: Cantor spectrum for Schrödinger operators with potentials arising from generalized skew-shifts. Duke Math. J. 146, 253-280 (2009) Zbl 1165.37012 MR 2477761

[AF] Avila, A., Forni, G.: Weak mixing for interval exchange transformations and translation flows. Ann. of Math. 165, 637-664 (2007) Zbl 1136.37003 MR 2299743

[AJ1] Avila, A., Jitomirskaya, S.: The Ten Martini Problem. Ann. of Math. 170, 303-342 (2009) Zbl 1166.47031 MR 2521117 
[AJ2] Avila, A., Jitomirskaya, S.: Almost localization and almost reducibility. J. Eur. Math. Soc. 12, 93-131 (2010) Zbl 1185.47028 MR 2578605

[AK] Avila, A., Krikorian, R.: Reducibility or nonuniform hyperbolicity for quasiperiodic Schrödinger cocycles. Ann. of Math. 164, 911-940 (2006) Zbl 1138.47033 MR 2259248

[AS1] Avron, J., Simon, B.: Almost periodic Schrödinger operators. I. Limit periodic potentials. Comm. Math. Phys. 82, 101-120 (1981) Zbl 0484.35069 MR 0638515

[AS2] Avron, J., Simon, B.: Almost periodic Schrödinger operators. II. The integrated density of states. Duke Math. J. 50, 369-391 (1983) Zbl 0544.35030 MR 0700145

[BCL] Béguin, F., Crovisier, S., Le Roux, F.: Construction of curious minimal uniquely ergodic homeomorphisms on manifolds: the Denjoy-Rees technique. Ann. Sci. École Norm. Sup. 40, 251-308 (2007) Zbl 1132.37003 MR 2339286

[Be1] Bellissard, J.: Schrödinger operators with almost periodic potential: An overview. In: Mathematical Problems in Theoretical Physics (Berlin, 1981), Lecture Notes in Phys. 153, Springer, Berlin, 356-363 (1982)

[Be2] Bellissard, J.: $K$-theory of $C^{*}$-algebras in solid state physics. In: Statistical Mechanics and Field Theory: Mathematical Aspects (Groningen, 1985), Lecture Notes in Phys. 257, Springer, Berlin, 99-156(1986) Zbl 0612.46066 MR 0862832

[Be3] Bellissard, J.: Gap labelling theorems for Schrödinger operators. In: From Number Theory to Physics (Les Houches, 1989), Springer, Berlin, 538-630 (1992) Zbl 0833.47056 MR 1221111

[Be4] Bellissard, J.: The noncommutative geometry of aperiodic solids. In: Geometric and Topological Methods for Quantum Field Theory (Villa de Leyva, 2001), World Sci., River Edge, NJ, 86-156 (2003) Zbl 1055.81034 MR 2009996

[BBG] Bellissard, J., Benedetti, R., Gambaudo, J.-M.: Spaces of tilings, finite telescopic approximations and gap-labeling. Comm. Math. Phys. 261, 1-41 (2006) Zbl 1116.46063 MR 2193205

[BLT] Bellissard, J., Lima, R., Testard, D.: Almost periodic Schrödinger operators. In: Mathematics + Physics. Vol. 1, World Sci., Singapore, 1-64 (1985) Zbl 0675.34022 MR 0849342

[BSc] Bellissard, J., Scoppola, E.: The density of states for almost periodic Schrödinger operators and the frequency module: a counterexample. Comm. Math. Phys. 85, 301-308 (1982) Zbl 0505.46056 MR 0676003

[BSi] Bellissard, J., Simon, B.: Cantor spectrum for the almost Mathieu equation. J. Funct. Anal. 48, 408-419 (1982) Zbl 0516.47018 MR 0678179

[BO] Benameur, M.-T., Oyono-Oyono, H.: Gap-labelling for quasi-crystals (proving a conjecture by J. Bellissard). In: Operator Algebras and Mathematical Physics (Constanţa, 2001), Theta, Bucharest, 11-22 (2003) MR 2018220

[BJ] Bjerklöv, K., Jäger, T.: Rotation numbers for quasiperiodically forced circle maps-modelocking vs. strict monotonicity. J. Amer. Math. Soc. 22, 353-362 (2009) Zbl 1206.37020 MR 2476777

[Bo] Bochi, J.: Genericity of zero Lyapunov exponents. Ergodic Theory Dynam. Systems 22, 1667-1696 (2002) Zbl 1023.37006 MR 1944399

[BC] Bonatti, C., Crovisier, S.: Récurrence et généricité. Invent. Math. 158, 33-104 (2004) Zbl 1071.37015 MR 2090361

[Ca] Cameron, R. H.: Linear differential equations with almost periodic coefficients. Ann. of Math. 37, 29-42 (1936) Zbl 0014.01703 MR 1503266

[CEY] Choi, M. D., Elliott, G. A., Yui, N.: Gauss polynomials and the rotation algebra. Invent. Math. 99, 225-246(1990) Zbl 0665.46051 MR 1031901 
[Ch] Chulaevskiŭ, V. A.: An inverse spectral problem for limit-periodic Schrödinger operators. Funktsional. Anal. i Prilozhen. 18, no. 3, 63-66 (1984) (in Russian) Zbl 0608.34025 MR 0757250

[CS] Chulaevskiŭ, V. A., Sină̌, Ya. G.: Anderson localization for the 1-D discrete Schrödinger operator with two-frequency potential. Comm. Math. Phys. 125, 91-112 (1989) Zbl 0743.60058

[CF] Cong, N. D., Fabbri, R.: On the spectrum of the one-dimensional Schrödinger operator. Discrete Contin. Dynam. Systems 9, 541-554 (2008) Zbl 1146.39027 MR 2379426

[Co] Connes, A.: An analogue of the Thom isomorphism for crossed products of a $C^{*}$-algebra by an action of $\mathbb{R}$. Adv. Math. 39, 31-55 (1981) Zbl 0461.46043 MR 0605351

[DS] Delyon, F., Souillard, B.: The rotation number for finite difference operators and its properties. Comm. Math. Phys. 89, 415-426 (1983) Zbl 0525.39003 MR 0709475

[E] Eliasson, L. H.: Floquet solutions for the 1-dimensional quasi-periodic Schrödinger equation. Comm. Math. Phys. 146, 447-482 (1992) Zbl 0753.34055 MR 1167299

[EJ] Ellis, R., Johnson, R.: Topological dynamics and linear differential systems. J. Differential Equations 44, 21-39 (1982) Zbl 0501.58033 MR 0651685

[FJ] Fabbri, R., Johnson, R.: Genericity of exponential dichotomy for two-dimensional differential systems. Ann. Mat. Pura Appl. 178, 175-193 (2000) Zbl 1037.34043 MR 1849385

[FJP] Fabbri, R., Johnson, R., Pavani, R.: On the nature of the spectrum of the quasiperiodic Schrödinger operator. Nonlinear Anal. Real World Appl. 3, 37-59 (2002) Zbl 1036.34097 MR 1941947

[Fa] Fayad, B.: Analytic mixing reparametrizations of irrational flows. Ergodic Theory Dynam. Systems 22, 437-468 (2002) Zbl 1136.37307 MR 1898799

[Fu] Furstenberg, H.: Strict ergodicity and transformation of the torus. Amer. J. Math. 83, 573601 (1961) Zbl 0178.38404 MR 0133429

[GS] Goldstein, M., Schlag, W.: On resonances and the formation of gaps in the spectrum of quasi-periodic Schrödinger equations. Ann. of Math. 173, 337-475 (2011) Zbl pre05960662 MR 2753606

[HS] Helffer, B., Sjöstrand, J.: Semiclassical analysis for Harper's equation. III. Cantor structure of the spectrum. Mém. Soc. Math. France 39 (1989), 124 pp. Zbl 0725.34099 MR 1041490

$[\mathrm{H}] \quad$ Herman, M.-R.: Une méthode pour minorer les exposants de Lyapounov et quelques exemples montrant le caractère local d'un théorème d'Arnol'd et de Moser sur le tore de dimension 2. Comment. Math. Helv. 58, 453-502 (1983) Zbl 0554.58034 MR 0727713

[J] Johnson, R.: Exponential dichotomy, rotation number, and linear differential operators with bounded coefficients. J. Differential Equations 61, 54-78 (1986) Zbl 0608.34056 MR 0818861

[JM] Johnson, R., Moser, J.: The rotation number for almost periodic potentials. Comm. Math. Phys. 84, 403-438 (1982) Zbl 0497.35026 MR 0714441

[KP] Kaminker, J., Putnam, I.: A proof of the gap labeling conjecture. Michigan Math. J. 51, 537-546 (2003) Zbl 1054.46047 MR 2021006

[KZ] Kellendonk, J., Zois, I.: Rotation numbers, boundary forces and gap labelling. J. Phys. A 38, 3937-3946 (2005) Zbl 1065.81057 MR 2144527

$[\mathrm{K}] \quad$ Knill, O.: The upper Lyapunov exponent of $\operatorname{SL}(2, \mathbb{R})$ cocycles: discontinuity and the problem of positivity. In: Lyapunov Exponents (Oberwolfach, 1990), Lecture Notes in Math. 1486, Springer, Berlin, 86-97 (1991) Zbl 0746.58050 MR 1178949

[L] Last, Y.: Zero measure spectrum for the almost Mathieu operator. Comm. Math. Phys. 164, 421-432 (1994) Zbl 0814.11040 MR 1289331 
[MMY] Marmi, S., Moussa, P., Yoccoz, J.-C.: The cohomological equation for Roth-type interval exchange maps. J. Amer. Math. Soc. 18, 823-872 (2005) Zbl 1112.37002 MR 2163864

[M] Moser, J.: An example of a Schrödinger equation with almost periodic potential and nowhere dense spectrum. Comment. Math. Helv. 56, 198-224 (1981) Zbl 0477.34018 MR 0630951

[N] Nirenberg, L.: On elliptic partial differential equations. Ann. Scuola Norm. Sup. Pisa 13, 115-162 (1959) Zbl 0088.07601 MR 0109940

[PT] A. Pastur, L., Tkachenko, V. A.: Spectral theory of a class of one-dimensional Schrödinger operators with limit-periodic potentials. Trans. Moscow Math. Soc. 1989, 115-166 Zbl 0709.34064 MR 0983634

[PV] Pimsner, M., Voiculescu, D.: Exact sequences for $K$-groups and Ext-groups of certain cross-product $C^{*}$-algebras. J. Operator Theory 4, 93-118 (1980) Zbl 0474.46059 MR 0587369

[Pa] Parry, W.: Topics in Ergodic Theory. Cambridge Univ. Press (1981) Zbl 0449.28016 MR 0614142

[Pu] Puig, J.: Cantor spectrum for the almost Mathieu operator. Comm. Math. Phys. 244, 297 309 (2004) Zbl 1075.39021 MR 2031032

[Sc] Schwartzman, S.: Asymptotic cycles. Ann. of Math. 66, 270-284 (1957) Zbl 0207.22603 MR 0088720

[Si1] Simon, B.: On the genericity of nonvanishing instability intervals in Hill's equation. Ann. Inst. H. Poincaré Sect. A 24, 91-93 (1976) Zbl 0346.34015 MR 0473321

[Si2] Simon, B.: Almost periodic Schrödinger operators: A review. Adv. Appl. Math. 3, 463490 (1982) Zbl 0545.34023 MR 0682631

[X] Xia, J.: The Floquet theory and the state density of quasi-periodic Schrödinger operators. J. Differential Equations 86, 243-259 (1990) Zbl 0714.34079 MR 1064013

[Y] Yoccoz, J.-C.: Some questions and remarks about $\operatorname{SL}(2, \mathbb{R})$ cocycles. In: Modern Dynamical Systems and Applications, Cambridge Univ. Press, Cambridge, 447-458 (2004) Zbl 1148.37306 MR 2093316 